
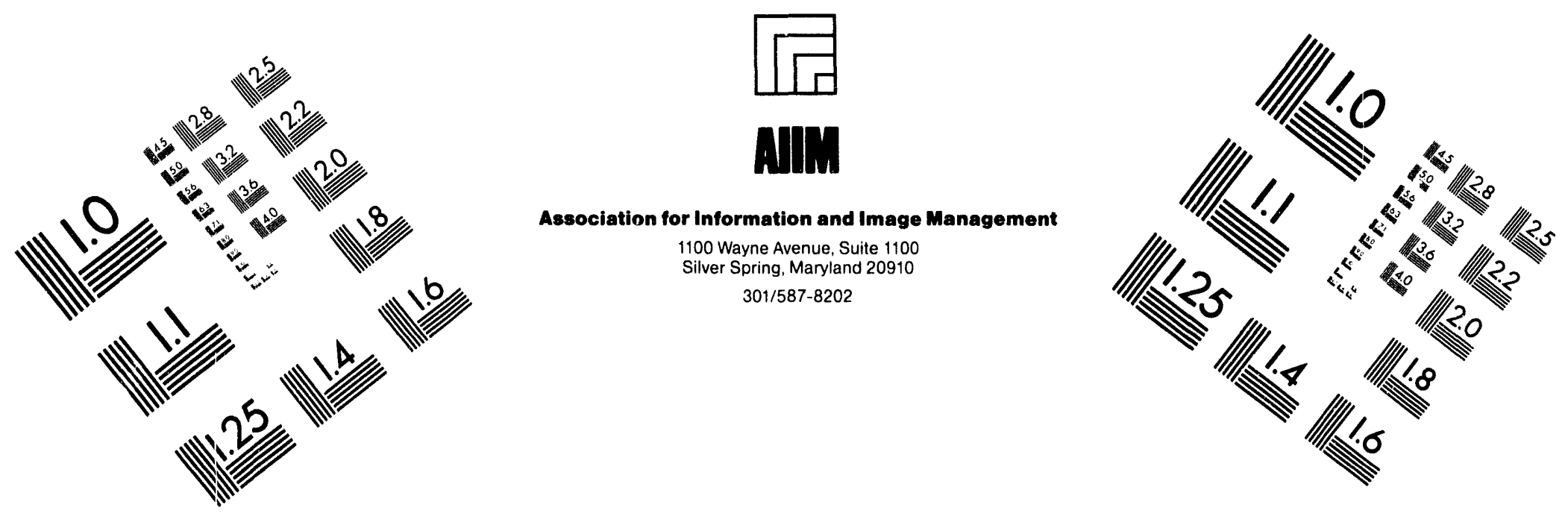

\title{
Centimeter
}

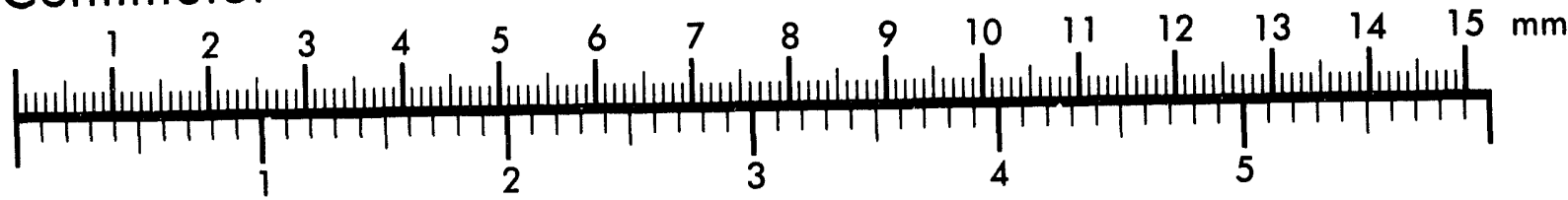

Inches
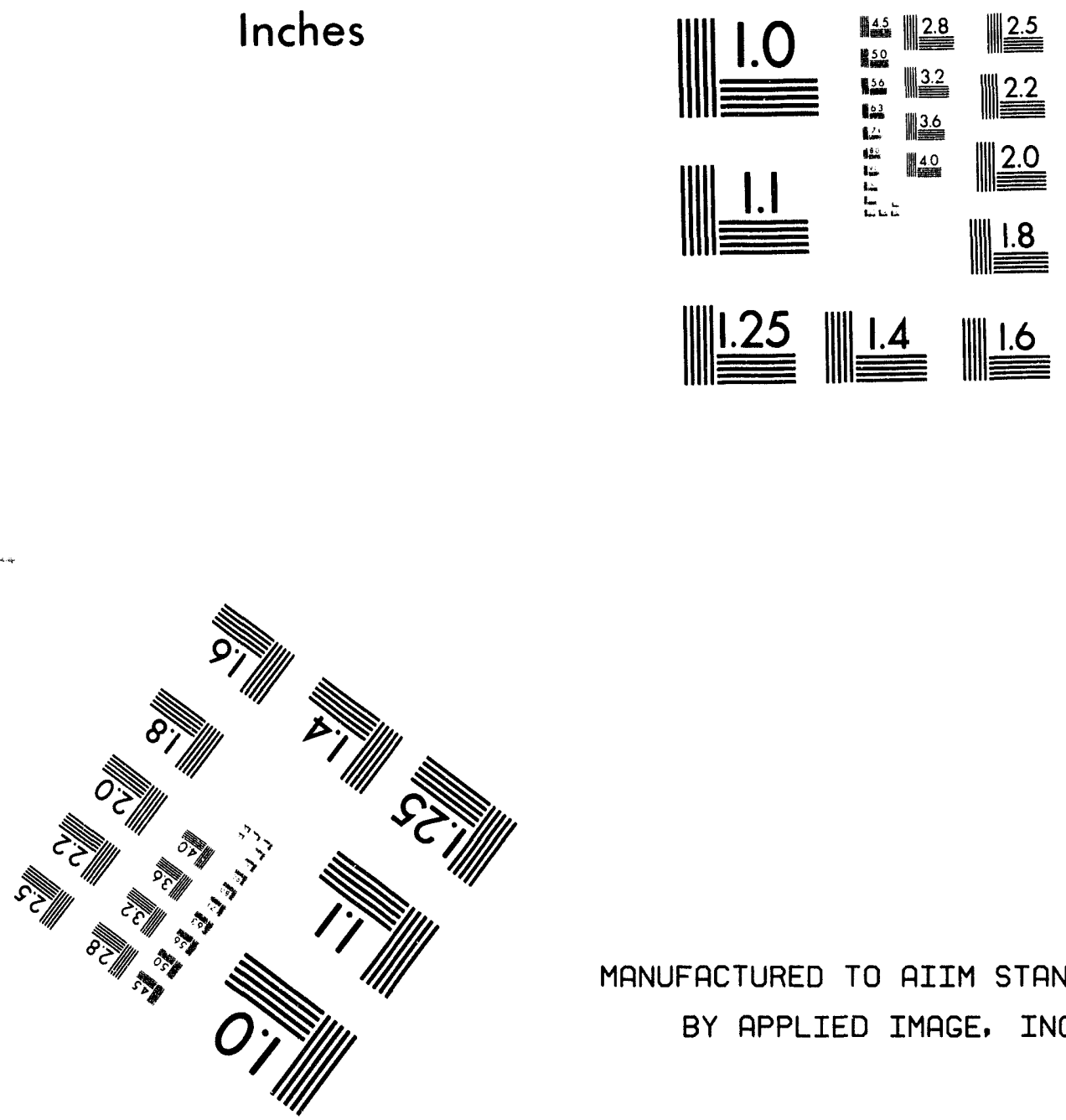

MANUFACTURED TO AIIM STANDARDS

BY APPLIED IMAGE, INC.

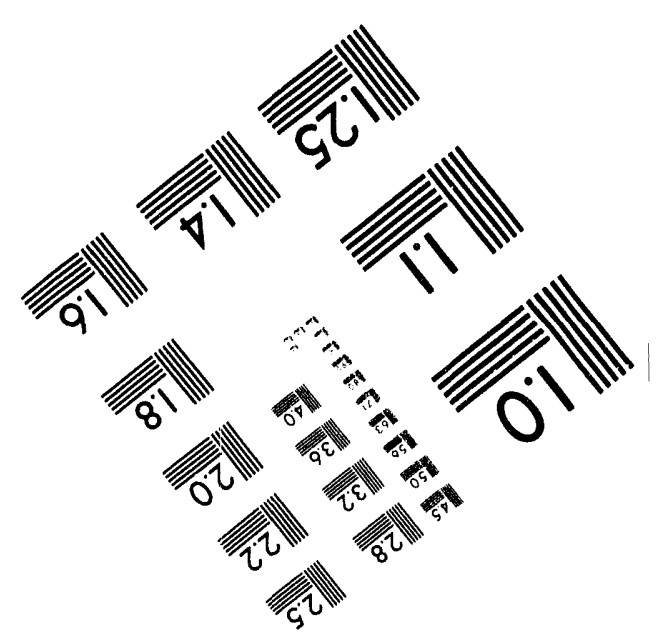



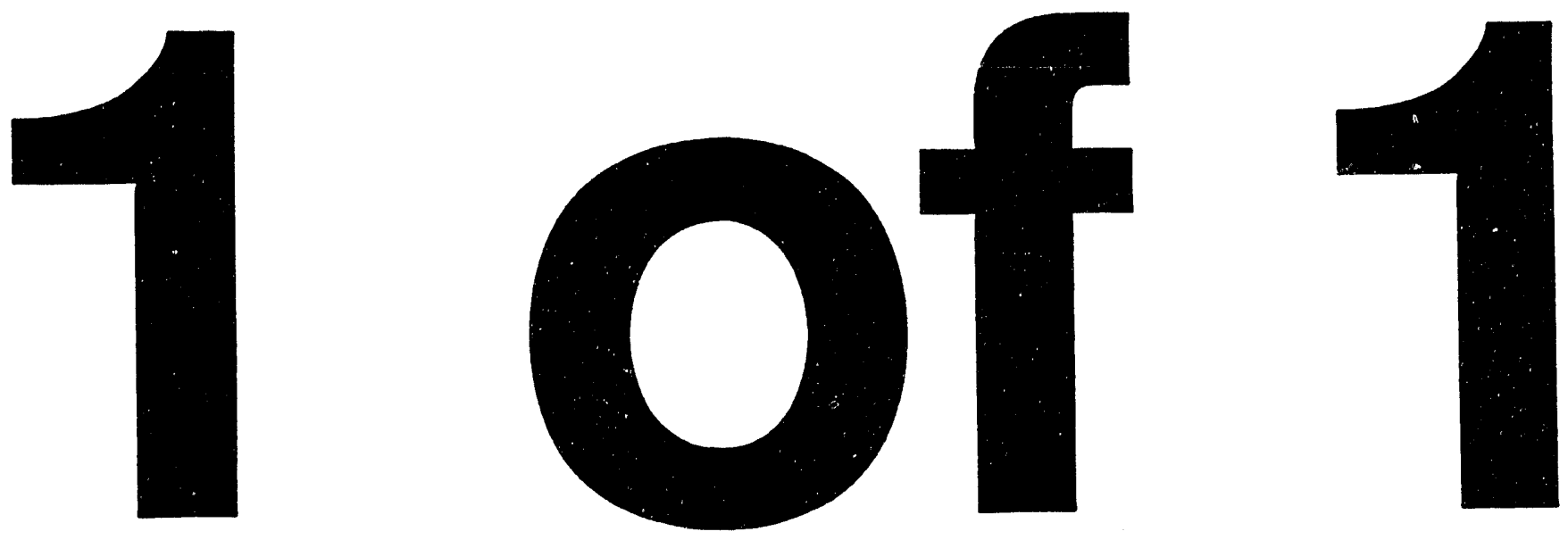
Metals and Ceramics Division

\section{PHASE AND GROUP VELOCITIES FOR LAMB WAVES IN DOP-26 IRIDIUM ALLOY SHEET}

W. A. Simpson and D. J. McGuire

Date Published: July 1994

\section{Prepared for}

U.S. Department of Energy

Office of Space and Defense Power Systems

Radioisotope Power Systems Division AF 7010200

Prepared by the

OAK RIDGE NATIONAL LABORATORY

Oak Ridge, Tennessee 37831-6080 managed by

MARTIN MARIETTA ENERGY SYSTEMS, INC. for the

U.S. DEPARTMENT OF ENERGY under contract DE-ACO5-84OR21400

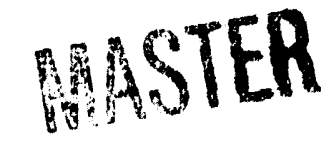




\section{CONTENTS}

\section{PAGE}

LIST OF FIGURES $\ldots \ldots \ldots \ldots \ldots \ldots \ldots \ldots \ldots \ldots \ldots \ldots, \quad \mathbf{v}$

ABSTRACT $\ldots \ldots \ldots \ldots \ldots \ldots \ldots \ldots \ldots \ldots \ldots \ldots \ldots \ldots \ldots \ldots \ldots \ldots \ldots \ldots, 1$

1. INTRODUCTION $\ldots \ldots \ldots \ldots \ldots \ldots \ldots \ldots \ldots \ldots \ldots \ldots \ldots \ldots \ldots \ldots \ldots \ldots$

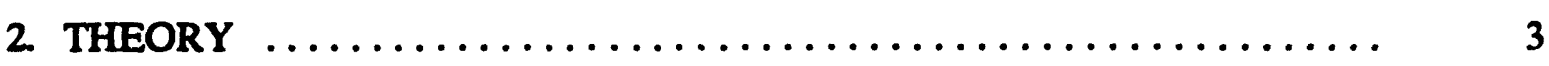

2.1 PHASE VELOCITY $\ldots \ldots \ldots \ldots \ldots \ldots \ldots \ldots \ldots \ldots \ldots \ldots \ldots \ldots \ldots, 3$

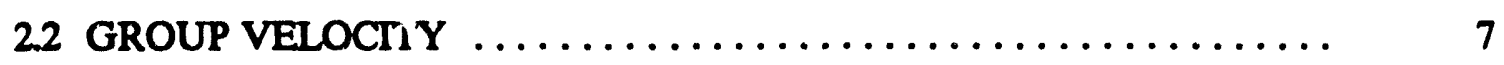

2.3 TIME-DOMAIN WAVEFORM $\ldots \ldots \ldots \ldots \ldots \ldots \ldots \ldots \ldots \ldots, 9$

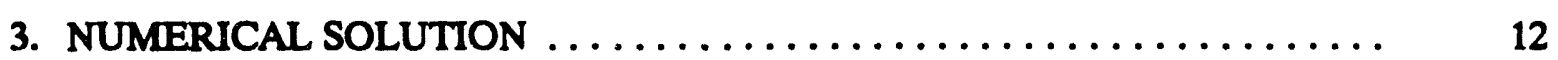

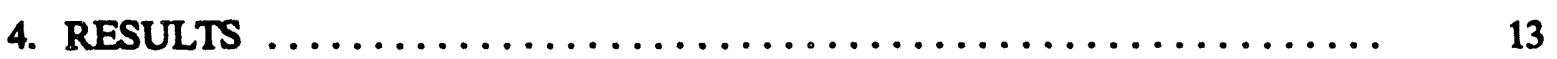

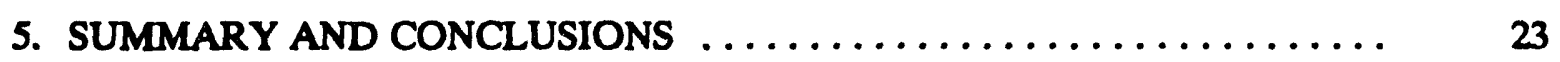

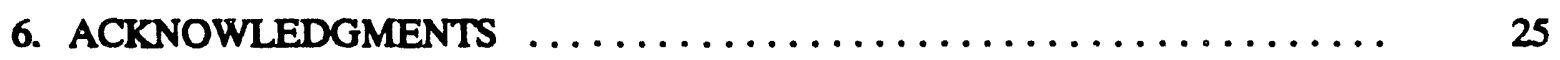

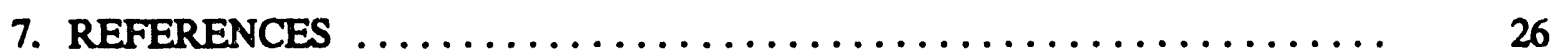




\section{LIST OF FIGURES}

PAGE

1 Geometry for Lamb wave propagation in a plate $\ldots \ldots \ldots \ldots \ldots \ldots \ldots$

2 Symmetric and antisymmetric distortions in a plate caused by

propagating Lamb wave $\ldots \ldots \ldots \ldots \ldots \ldots \ldots \ldots \ldots \ldots \ldots \ldots \ldots \ldots \ldots \ldots$

3 Phase velocity dispersion curves showing Lamb wave modes in

iridium alloy $\ldots \ldots \ldots \ldots \ldots \ldots \ldots \ldots \ldots \ldots \ldots \ldots \ldots \ldots \ldots \ldots \ldots \ldots$

4 Group velocity dispersion curves showing Lamb wave modes in

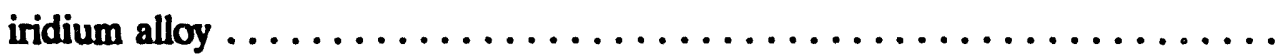

5 Radio frequency waveform of transducers used to excite Lamb waves in iridium plate alloy

6 Spectrum of transducers used to excite Lamb waves in an iridium plate alloy

7 Radio frequency waveform of $A_{0}$ Lamb mode in iridium alloy at $39^{\circ}$ incident angle $\ldots \ldots \ldots \ldots \ldots \ldots \ldots \ldots \ldots \ldots \ldots \ldots \ldots \ldots \ldots$

8 Spectrum of $A_{0}$ Lamb mode in iridium alloy at $39^{\circ}$ incident angle . . . . . .

9 Radio frequency waveform of combined $S_{0}, A_{1}$, and $S_{1}$ Lamb modes in iridium alloy at $18.5^{\circ}$ incident angle $\ldots \ldots \ldots \ldots \ldots \ldots \ldots$

10 Spectrum of $S_{0}, A_{1}$, and $S_{1}$ Lamb modes in iridium alloy at $18.5^{\circ}$ incident angle.

11 Radio frequency waveform of combined $A_{1}, S_{1}, A_{2}$ and $S_{2}$ Lamb 11odes in iridium alloy at $11^{\circ}$ incident angle $\ldots \ldots \ldots \ldots \ldots \ldots$

12 Spectrum of $A_{1}, S_{1}, A_{2}$ and $S_{2} L$, nb modes in iridium alloy at $11^{\circ}$ incident angle.

13 Measured values of group velocity for four Lamb modes in iridium alloy 


\title{
PHASE AND GROUP VELOCITIES FOR LAMB WAVES IN DOP-26 IRIDIUM ALLOY SHEET*
}

\author{
W. A. Simpson, Jr., and D. J. McGuire
}

\begin{abstract}
The relatively coarse grain structure of iridium weldments limits the ultrasonic inspection of these structures to frequencies in the low megahertz range. As the material thickness is nominally $0.635 \mathrm{~mm}$ for clad vent set capsules, the low frequencies involved necessarily entail the generation of Lamb waves in the specimen. These waves are, of course, dispersive, and detailed knowledge of both the phase and group velocities is required in order to determine accurately the location of flaws detected using Lamb waves. The purpose of this study is to elucidate the behavior of Lamb waves propagating in the capsule alloy and to quantify the velocities so that accurate flaw location is ensured. We describe a numerical technique for computing the phase velocities of Lamb waves (or of any other type of guided wave) and derive the group velocities from this information. A frequency-domain method is described for measuring group velocity when multiple Lamb modes are present and mutually interfering in the time domain, and experimental confirmation of the group velocity is presented for the capsule material.
\end{abstract}

\section{INTRODUCTION}

DOP-26 iridium-base alloy containing nominally $0.3 \mathrm{wt} \%$ tungsten, $60 \mathrm{wppm}$ thorium, and 50 wppm aluminum was developed at Oak Ridge National Laboratory (ORNL) for cladding plutonium oxide pellets in radioisotope thermoelectric generators. Although it has performed well in the intended application, the severe elastic wave scattering from the relatively large grain size inherent in weldments of this alloy apparently limits the maximum frequency for ultrasonic inspection of such weldments to a few megahertz. I'nfortunately, if the specimen is Iso thin (i.e., comparable to a few wavelengths or less), then the resulting waves will interact with both boundary surfaces simultaneously, and the infinite-medium propagation assumption of

\footnotetext{
* Research sponsored by the Office of Space and Defense Power Systems, Radioisotope Power Systems Division, U.S. Department: of Energy, Office of Nuclear Energy, under contract DE-AC05-84OR21400 with Martin Marietta Energy Systems, Inc.
} 
conventional ultrasonic evaluation is no longer valid. For example, the total temporal extent of even a highly damped ultrasonic pulse will be about three cycles of the center frequency, or about 0.6 Hs at $5 \mathrm{MHz}$. In iridium alloy (longitudinal wave velocity $5.035 \mathrm{~km} / \mathrm{s}$ ), this produces a wave train more than $3 \mathrm{~mm}$ long. For the 0.635 -mm-thick (less than one wavelength at $5 \mathrm{MHz}$ ) DOP-26 iridium alloy clad vent set capsules, this means that the ultrasonic energy will interact with both bounding surfaces of the specimen simultaneously. Under these conditions, nondispersive bulk-wave propagation is replaced by a fundamentally different form of elastic guided wave propagation, i.e., by Lamb wave propagation.

Since Lamb waves are dispersive (both phase and group velocities are functions of frequency), it is imperative that the ultrasonic investigator have a clear understanding of this aspect of their behavior. In particular, if measurements are to be performed in the time domain, it is the behavior of the group velocity that is of interest, since this is the quantity actually measured. As in the case of electromagnetic wave propagation, the group velocity of Lamb waves may either exceed the phase velocity (normal dispersion) or be less than the phase velocity (anomalous dispersion). In addition, the occurrence of negative group velocities has recently been theoretically predicted and experimentally confirmed for Lamb waves in certain materials. ${ }^{1}$ This phenomenon, which leads to the decidedly nonintuitive condition in which energy propagates counter to the phase velocity (i.e., the wave propagates backwards), has apparently not been observed in the electromagnetic case. The strange nature of elastodynamic wave propagation in thin structures underscores the need to have a strong theoretical understanding of elastic guided wave propagation.

The behavior of elastic wave propagation in plates whose thickness approximates a wavelength was first elucidated by Lamb, ${ }^{2}$ who showed that the energy is distributed among a series of modes which are analogous to the transverse electric (TE) and transverse magnetic (TM) modes of electromagnetic waves in waveguides. A recent, excellent treatment of Lamb wave propagation has been given by Achenbach, ${ }^{3}$ who derives the familiar transcendental equation connecting phase velocity and wave vector.

Our approach in developing a solution for Lamb waves was somewhat different. Several years ago, we became interested in the general problem of the propagation of guided elastic waves of all types. This interest was engendered by the observation that the velocity of guided elastic waves is very sensitive to the boundary conditions at a solid-solid interface, and, hence, such waves might be an excellent tool for assessing nondestructively the strength of solid-solid 
joints. In particular, we were interested in guided waves in the center layer of a three-layer solid, a problem that had apparently not been solved previously. After obtaining a general solution for the three-layer case, we realized that by restricting the secular determinant to the order appropriate to other waves in layered structures, the anatytical and numerical techniques that we had developed to solve the three-layer problem could be used to compute results for all of the other common types of guided waves, such as Rayleigh, Lamb, Stoneley, and Love waves, as well as for "leaky" waves of each of these types. Leaky waves are guided waves which "leak" energy into the surrounding layers and correspond to solutions of the secular determinant for which the wave vector is complex. These techniques have been used in the present case to investigate the properties of Lamb waves propagating in iridium alloy sheet.

Strictly speaking, the waves excited in the inspection of iridium alloy capsules are leaky Lamb waves, and the properties of such waves can be quite different from those of the true (nonleaky) Lamb wave.5 However, when the acoustic impedance of the material in which the waves propagate differs widely from that of the surrounding medium, the difference between guided and leaky waves is negligible. For example, the phase velocity of a leaky Rayleigh wave on iron differs from the true Rayleigh wave by only about $0.05 \%$, assuming that water is the medium into which the wave leaks. In the case of iridium alloy, the acoustic impedance disparity is even greater, and, hence, the difference in the leaky and nonleaky Lamb wave velocities will be still less.

Our work in guided elastic waves, "although encompassing both leaky and true Lamb waves, did not explicitly present the secular determinant for these cases. Accordingly, we shall present the theoretical derivation of the equations of propagation for Lamb waves before discussing the numerical solution of the resulting secular determinant.

\section{THEORY}

\section{PHASE VEIOCTTY}

Figure 1 shows the geometry for the Lamb wave problem. The wave is a umed to have no $y$ dependence and to propagate in the $x$ direction in a layer of thickness $2 \mathrm{~b}$. The layer 


\section{ORNL-DWG 94-10473}

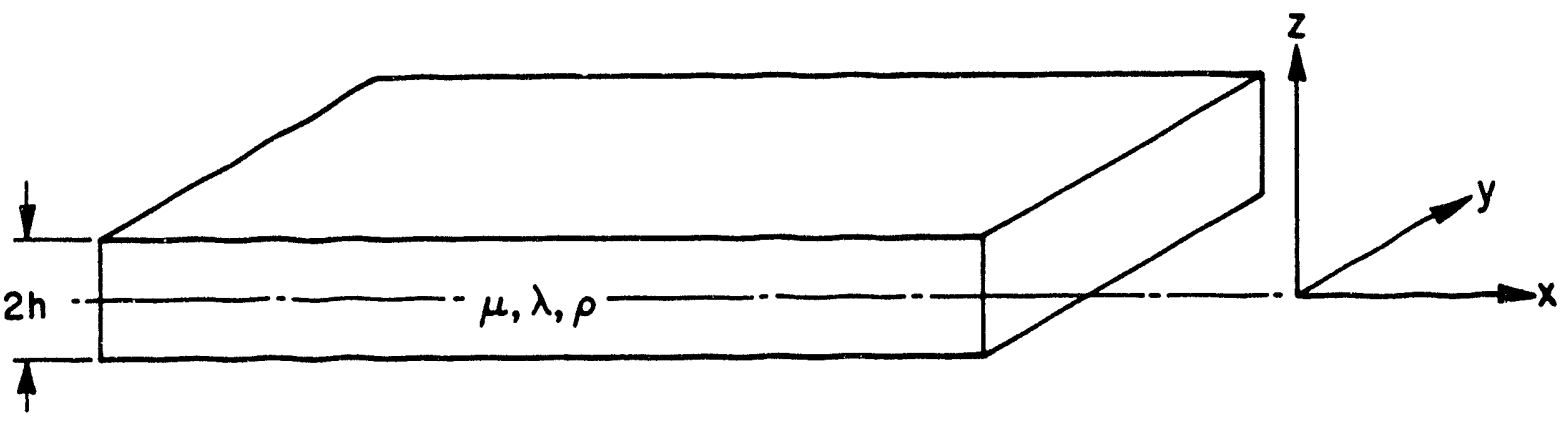

Fig. 1. Geometry for Lamb wave propagation in a plate.

material is assumed to be linear and isotropic with elastic constants $\mu$ and $\lambda$. The equation of motion which must be satisfied in the plate is: ${ }^{6}$

$$
\mu \nabla^{2} \vec{S}+(\lambda+\mu) \nabla(\nabla \cdot \vec{S})=\rho \partial^{2} \vec{S} / \hat{t}^{2},
$$

where $\overrightarrow{\vec{S}}$ is the particle displacement, and $\rho$ is the material density. Now $\overrightarrow{\mathbf{s}}$ can be defined in terms of the usual potentials:

$$
\vec{S}=\nabla \phi+\nabla \times \vec{\Psi} .
$$

The displacement contains irrotational and solenoidal potentials that give rise to compressional and shear waves, respectively. Lamb waves, like all finite-layer guided waves, are thus seen to be very specific coupled sets of the usual bulk waves.

Substituting Eq. (2) into Eq. (1), we see that $\phi$ and $\vec{\nabla}$ satisfy:

$$
\nabla^{2} \phi=\left(1 / C_{l}^{2}\right) \partial^{2} \Phi / \partial^{2}, \quad \nabla^{2} \Psi=\left(1 / C_{l}^{2}\right) \partial^{2} \Psi / \partial^{2},
$$

where $C_{1}$ is the longitudinal wave velocity and $C_{1}$ the shear wave velocity in the plate. We seek solutions whose $x$ dependence is of the form exp(ikx) and which are independent of $y$. The latter condition requires that $\vec{\psi}=\left(0, \Psi_{y}, 0\right)$; we therefore let $\Psi_{y}=\psi$. We also assume that the solutions are time harmonic of the form $\exp (-i \omega t)$, where $\omega$ is the angular frequency. Thus:

$$
\nabla^{2} \phi=-\omega^{2} \phi / C_{l}^{2}=-k_{l}^{2} \phi, \quad \nabla^{2} \psi=-k_{l}^{2} \psi,
$$

or $\left(\nabla^{2}+k_{1}^{2}\right) \phi=0$ and $\left(\nabla^{2}+k_{1}^{2}\right) \psi=0$. Now:

$$
\nabla^{2}=\partial^{2} / \partial x^{2}+\partial^{2} / \partial z^{2}=-k^{2}+\partial^{2} / \partial z^{2} .
$$

Thus, the potentials satisfy:

$$
d^{2} \phi / d z^{2}=\alpha^{2} \phi, \quad d^{2} \Psi / d z^{2}=\beta^{2} \psi,
$$


where $\alpha^{2}=k^{2}-k_{1}^{2}$ and $\beta^{2}=k^{2}-k_{1}^{2}$. The solutions to these equations are of the form:

$$
\phi=A \exp ( \pm \alpha z) \exp [i(k x-\omega t)], \quad=B \exp ( \pm \beta z) \exp [i(k x-\omega t)] .
$$

where $A$ and $B$ are arbitrary coefficients.

The displacement $\vec{S}$ from Eq. (2) is given by:

$$
S_{x}=\partial \phi / \partial x-\partial \psi / \partial z, \quad S_{z}=\partial \phi / 2 z+\partial \psi / \partial x \text {. }
$$

The stresses are calculated from the stress-strain relationship in an isotropic solid:

$$
\sigma_{i j}=\lambda \delta_{j} \varepsilon_{k k}+2 \mu \varepsilon_{j j} \text {. }
$$

where

$$
e_{y j}=\left(\partial S_{i} / \partial x_{j}+2 S_{j} / \partial x_{j}\right) / 2
$$

is the strain tensor, and $\varepsilon_{k \mathrm{k}}$, the trace of the strain tensor, is the dilatation. The term $\delta_{\mathrm{ij}}$ is the Kronecker delta function. Substituting for $S_{k}$ above gives:

$$
\begin{gathered}
\sigma_{z z}=\lambda\left(\partial^{2} \phi / \partial x^{2}+\partial^{2} \phi / \partial z^{2}\right)+2 \mu\left(\partial^{2} \phi / \partial z^{2}+\partial^{2} \psi / \partial z \partial x\right) \\
=-\lambda k_{1}^{2} \phi+2 \mu \alpha^{2} \phi+2 \mu i k \partial \psi / \partial z .
\end{gathered}
$$

However, $\alpha^{2}=k^{2}-k_{f}^{2}$. Thus:

$$
\sigma_{z z}=-(\lambda+2 \mu) k_{l}^{2} \phi+2 \mu k^{2} \phi+2 \mu i k \partial \psi / \partial z \text {. }
$$

Also, $(\lambda+2 \mu) k_{i}^{2}=\rho \omega^{2}=\mu k_{l}^{2}=\mu\left(k^{2}-\beta^{2}\right)$. Therefore:

$$
\sigma_{2 z} / \mu=\left(k^{2}+\beta^{2}\right) \phi+2 i k \partial \psi / \alpha z \text {. }
$$

Likewise:

$$
\sigma_{x z}=\mu\left(\partial S_{x} / \partial z+\partial S_{z} / \partial x\right)=\mu\left[2 \partial^{2} \phi / \partial x \partial z-\partial^{2} \psi / \partial z^{2}+\partial^{2} \Psi / \partial x^{2}\right],
$$

or

$$
\sigma_{x z} / \mu=2 i k \partial \phi / \partial z-\left(k^{2}+\beta^{2}\right) \psi
$$

Now that we have the displacements and stresses in terms of the displacement potentials, the solution for waves propagating in the plate can be written down straightforwardly. Since we have a single medium of finite thickness, the displacement potentials are:

$$
\begin{aligned}
& \phi=A_{1} \cos \alpha z+B_{1} \sin \alpha z, \\
& \psi=A_{2} \cos \beta z+B_{2} \sin \beta z, \quad|z|<h,
\end{aligned}
$$


where $A_{1}, B_{1}, A_{2}$ and $B_{2}$ are arbitrary amplitudes to be determined. The choice of circular sines and cosines for the displacement potentials was made to elucidate the symmetric and antisymmetric properties of Lamb waves, but this choice of potentials requires that the terms $\left(k^{2}+\beta^{2}\right)$ in Eqs. (8) and (9) be replaced by $\left(k^{2}-\beta^{2}\right)$.

The boundary conditions that must be satisfied at $z= \pm h$ are the vanishing of the normal and tangential stresses (traction-free surface). Calculating these values from Eqs. (8) and (9), we find that:

$$
\begin{aligned}
\left(k^{2}-\beta^{2}\right) A_{1} \cos \alpha h+\left(k^{2}-\beta^{2}\right) B_{1} \sin \alpha h-2 i k \beta A_{2} \sin \beta h+2 i k \beta B_{2} \cos \beta h & =0 \\
-2 i k \alpha A_{1} \sin \alpha h+2 i k \alpha B_{1} \cos \alpha h-\left(k^{2}-\beta^{2}\right) A_{2} \cos \beta h-\left(k^{2}-\beta^{2}\right) B_{2} \sin \beta h & =0 \\
\left(k^{2}-\beta^{2}\right) A_{1} \cos \alpha h-\left(k^{2}-\beta^{2}\right) B_{1} \sin \alpha h+2 i k \beta A_{2} \sin \beta h+2 i k \beta B_{2} \cos \beta h & =0 \\
2 i k \alpha A_{1} \sin \alpha h+2 i k \alpha B_{1} \cos \alpha h-\left(k^{2}-\beta^{2}\right) A_{2} \cos \beta h+\left(k^{2}-\beta^{2}\right) B_{2} \sin \beta h & =0 .
\end{aligned}
$$

If there is to be a nontrivial solution for this set of equations, then:

$$
\left|\begin{array}{cccc}
\left(k^{2}-\beta^{2}\right) \cos \alpha h & \left(k^{2}-\beta^{2}\right) \sin \alpha h & -2 i k \beta \sin \beta h & 2 i k \beta \cos \beta h \\
-2 i k \alpha \sin \alpha h & 2 i k \alpha \cos \alpha h & -\left(k^{2}-\beta^{2}\right) \cos \beta h & -\left(k^{2}-\beta^{2}\right) \sin \beta h \\
\left(k^{2}-\beta^{2}\right) \cos \alpha h & -\left(k^{2}-\beta^{2}\right) \sin \alpha h & 2 i k \beta \sin \beta h & 2 i k \beta \cos \beta h \\
2 i k \alpha \sin \alpha h & 2 i k \alpha \cos \alpha h & -\left(k^{2}-\beta^{2}\right) \cos \beta h & \left(k^{2}-\beta^{2}\right) \sin \beta h
\end{array}\right|=0 .
$$

Values of $\mathbf{k}$ which satisfy this determinantal equation yield the phase velocities as a function of frequency (dispersion relation) for each of the various Lamb modes.

After the roots of the secular determinant are found, one would also like to know the amplitudes of the various waves (i.e., the coefficients $A_{i}$ and $B_{i}$ for each potential). The coefficients are determined by solving the homogeneous matrix equation:

$$
\bar{D} \bar{Y}=0 \text {, }
$$

where $\bar{D}$ is the determinant matrix, and $\bar{Y}$ is the coefficient matrix. Since the equation is homogeneous, one can only solve for ratios of coefficients. Thus, we set one of the coefficients to unity and solve for the others in terms of the prescribed amplitude. When this is done, two classes of waves, designated symmetric and antisymmetric because of the distortions induced in the plate as the wave propagates (see Fig. 2), are found. In our case, we first set the coefficient of the cosine term, $A_{b}$ in the shear potential to unity and solve for the other coefficients. For an antisymmetric wave, we shall then find that $\mathrm{B}_{2}$ is negligible (the displacement is the derivative 
ORNLDWG 86-1861

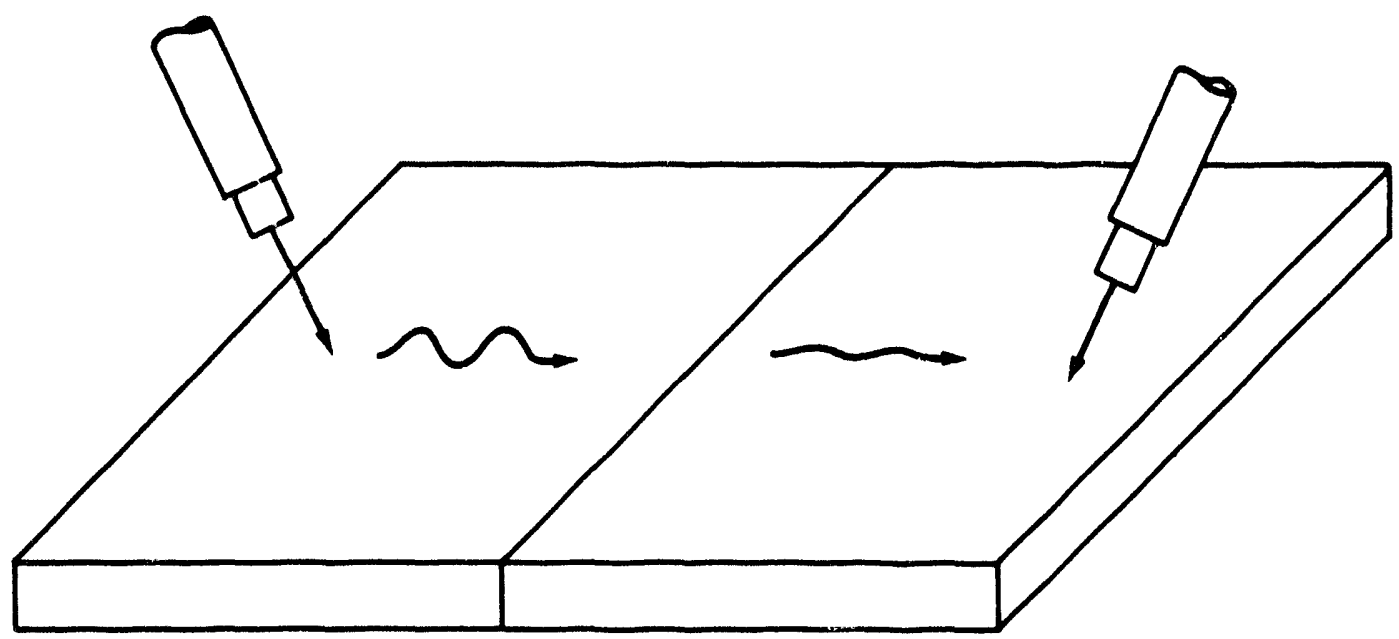

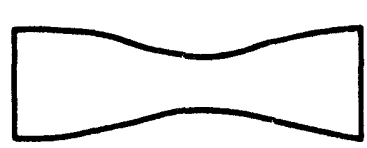

SYMMETRIC

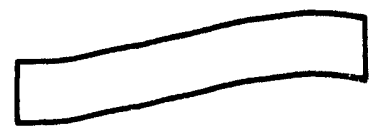

ANTISYMMETRIC

Fig. 2. Symmetric and antisymmetric distortions in a plate caused by propagating Lamb wave.

of its associated potential). If the wave is symmetric, however, the value for $B_{2}$ will be very large, and we repeat the process with $B_{2}$ set to unity.

\section{GROUP VELOCITY}

Once the phase velocities have been determined, the group velocities can be calculated as follows. By definition, the group velocity is:

$$
V_{s}=d \omega / d k \text {. }
$$

However, the phase velocity, $c$, is given by:

$$
\omega=c k \text {. }
$$

Taking the total derivative of both sides of this equation and dividing by dw:

$$
1=c d k / d \omega+k d c / d \omega=c / V_{8}+k d c / d \omega=\frac{c}{V_{8}}+\frac{v}{c} d c / d v,
$$


where $v$ is the frequency. Solving for $V_{g}$ :

$$
V_{2}=\frac{c^{2}}{c-v d c / d v}
$$

Thus, once we have determined the phase velocity as a function of frequency, the group velocity can be calculated from the slope of the dispersion curve (dc/dv) at any given frequency using Eq. (16).

The measurement of Lamb wave group velocity is notoriously difficult when more than one mode is present concurrently. Each mode contributes its own rf (radio frequency) wave packet, which combines with any others present to yield a very complex waveform. Examples of this phenomenon will be seen shortly in the section on results. The contributions of each component to the composite if waveform often cannot be separated in the time domain unless the group velacities are sufficiently different, and the propagation distance is such that the various wave packets no longer overlap. In the frequency domain, however, the various modes that may be present are often distinct, and an accurate measurement of group velocity may be obtained. The measurement is effected by computing the fast Fourier transform (FFT) of the composite waveform, determining its phase spectrum, and computing the slope of this spectrum in the vicinity of the spectral peaks of the various waves. The phase slope is related to the group velocity as follows. The phase characteristic of a propagating plane wave is given by:

$$
\phi(\omega)=k x-\omega t .
$$

Differentiating with respect to $\omega$ :

$$
\frac{d \phi(\omega)}{d \omega}=x \frac{d k}{d \omega}-t=x / V_{2}-t
$$

Thus:

$$
V_{s}=x\left(\frac{d \phi(\omega)}{d \omega}+t\right)^{-1}
$$

The term $t$ in the denominator is an arbitrary time offset which may be set equal to zero here, provided that all measurements are made in a single digital window. It is also important to note that the phase spectrum is meaningful only in those frequency regions for which the amplitude spectrum is sensibly different from zero; we chose the spectral maxima to ensure good signal-tonoise characteristics. 


\section{TIME-LOMAN WAVEFORM}

A useful adjunct to the determination of Lamb wave phase and group velocities would be the ability to predict the time-domain waveform of a single mode, or of combinations of modes, as a function of the propagation distance. Since the waves are dispersive, the shape of a wave packet will change with time (or distance) because of the different group velocities with which the various components of the packet propagate. Determination of the waveform for actual Lamb waves is a very difficult problem that requires numerical integration of the results obtained from the phase velocity studies and which depends strongly on the transducer characteristics and the specimen configuration. For example, the time-domain results observed for ultrasound introduced in the knuckle region of a capsule will differ considerably from those obtained on flat specincens, even when the same transducer is used, because the former condition encompasses a much greater range of incident angles and thus a potentially greater range of frequencies. In other words, the specific attributes of a particular test configuration must be combinel with the phase velocity results in order to predict accurately the time-domain response of Lamb waves.

Although a thorough study of the time-domain behavior of Lamb waves in the present case would require much more effort than we coi id afford to expend, some idea of the approach taken and of the results which can be obtained for a simple illustrative example can be provided. We shall see shortly that the if waveform of the $A_{0}$ Lamb mode can be modeled fairly accurately by a simple cosine-modulated Gaussian. If we can also assume that linear superposition holds for this case, make some simplifying assumptions about the functional relationship connecting $\omega$ and $k$, and neglect the z-dependence of the wave, the time-domain response can be determined analytically. Strictly speaking, ignoring the z-dependence of the wave is not necessary for signals sufficiently narrowband that the variation over plate thickness can be neglected. However, this will likely not be the case in practice, and as we will not present a full treatment of the timedomain solution, reducing the problem to a single dimension for illustrative purposes is not particularly restrictive. It merely indicates that we cannot solve the full problem analytically and must resort to numerical techniques.

Following the development of Jackson, ${ }^{8}$ we write the time-domain response of an actual (finite bandwidth) Lamb mode as a linear superposition of plane waves:

$$
u(x, t)=\frac{1}{2}\left[\int_{-\infty}^{-} A(k) e^{i[k x-\omega(x) t]} d k+c . c\right],
$$


where c.c indicates the complex conjugate, and where $\omega(k)$ indicates the functional relationship connecting angular frequency and wave number. In an actual case, this relationship would be taken from the phase velocity results obtained earlier and the integrals determined numerically. We also restrict ourselves to the case of true Lamb waves ( $k$ is real) so that there are no dissipative losses. It is understood that the actual if waveform is the real part of Eq. (20) and that the factor $(2 \pi)^{-1 / 2}$ in front of the integral, inserted by some authors to emphasize the Fourier transform relationship between $A(k)$ and $u(x, t)$, has been omitted.

Since our solutions were developed from a second-order differential equation, the initial conditions must specify both $u(x, 0)$ and its derivative. Multiplying Eq. $(20)$ by $\mathrm{e}^{-\mathrm{ikx}}$, integrating over all space, and using the completeness properties of the exponential:

$$
\int_{-\infty}^{\infty} u(x, 0) e^{-1 k x} d x=\pi\left[A(k)+A^{*}(-k)\right],
$$

where the asterisk indicates the complex conjugate. Taking the derivative of Eq. (20) and integrating over all space:

$$
\left.\int_{-\infty}^{\infty} \frac{\partial u}{\partial t}(x, 0) e^{-i k x} d x=\pi l(k)[-i \omega(k)]+A^{*}(-k)[i \omega(-k)]\right\}
$$

Since the properties of the wave cannot depend on whether it propagates in the positive or negative $x$-direction, $\omega(k)=\omega(-k)$; i.e., $\omega$ is an even function of $k$. Solving Eq. (21) and Eq. (22):

$$
A(k)=\frac{1}{2 \pi} \int_{-\infty}^{\infty} e^{-i k x}\left[u(x, 0)+\frac{i}{\omega(k)} \frac{\partial u}{\partial t}(x, 0)\right] d x .
$$

Equation (23) gives the Fourier amplitude of the pulse which, when substituted back into Eq. (20), yields the pulse shape as a function of time.

In order to obtain numerical results for the pulse shape, it is necessary to assume a functional relationship for $\omega(\mathbf{k})$. Since the function must be even, a simple relationship is:

$$
\omega(k)=\omega_{0}\left(1+\frac{a^{2} k^{2}}{2}\right),
$$

where $a$ has the dimensions of length and, as will be seen shortly, can be chosen to control the rate at which the pulse "spreads" in time. 
In addition to the functional relationship connecting $\omega$ and $k$, it is also necessary to specify the initial conditions. As mentioned earlier, a good approximation to the waveform of the $A_{0}$ mode is a cosine-modulated Gaussian. Accordingly, for the initial spatial domain conditions, we chose:

$$
\begin{gathered}
u(x, 0)=e^{-x^{2} / 2 \nabla^{2} \cos k_{0} x} \\
\frac{\partial u}{\partial t}(x, 0)=0,
\end{gathered}
$$

where $W$ is a characteristic width that controls the spatial extent of the pulse, and $k_{0}$ is the wave vector of the pulse frequency $\omega_{0}$. Substituting these expressions into Eq. (23) and performing the integration, we find for the Fourier amplitudes:

$$
A(k)=\frac{1}{\sqrt{2 \pi}} \frac{W}{2}\left[e^{-\left(W^{2} / 2\right)\left(k-k_{0}\right)^{2}}+e^{-\left(W^{2} / 2\right)\left(k+k^{2}\right)^{2}}\right] .
$$

We now substitute this expression and that given in Eq. (24) for $\omega(\mathrm{k})$ into Eq. (20) to find the time-domain behavior of our wave. The result is:

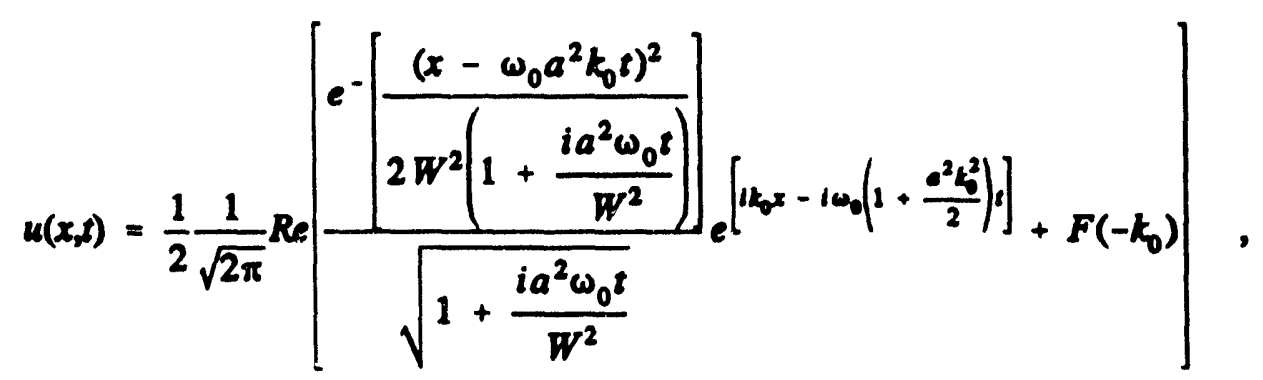

where $F\left(-k_{0}\right)$ represents the first part of Eq. (27) with $k_{0}$ replaced by $-k_{0}$. From Eq. (25), the initial width of the Gaussian envelope is W. Equation (27) (after first computing the real part of the complex exponential) shows that, at some later time $t$, the envelope width is given by:

$$
W(t)=\sqrt{W^{2}+\left(\frac{a^{2} \omega_{0} t}{W}\right)^{2}}
$$

On the other hand, the square root term in Eq. (27) shows that the pulse amplitude decreases with time. Thus, the pulse decays in amplitude and "spreads" in spatial extent as it propagates. This is precisely the behavior exhibited on the oscilloscope by the $A_{0}$ rf waveform (or by any other single Lamb mode).

Equation (28) also shows that the rate at which the pulse spreads is governed by the ratio $a^{2} / W$, which is the reason for our earlier statement that $a$ determines the rate of spreading, once an initial Gaussian width, W, has been chosen. 
Although the result contained within Eq. (28) is not directly applicable to the time-domain behavior of Lamb modes, it does illustrate the general properties of dispersive wave propagation and actually models rather well the behavior of certain modes. For actual Lamb waves, however, the two-dimensional solutions for the displacement obtained earlier would have to be integrated numerically using the functional relationship between $\omega$ and $\mathbf{k}$ implicit in the phase velocity results. While certainly possible, this would involve considerably more effort than we could afford to expend in this limited study. We have also not addressed the time-domain problem when more than one Lamb mode is present simultaneously. This would be a minor complication, however, since one could obtain solutions comparable to Eq. (28) for each mode and then coherently sum these time-domain results.

\section{NUMERICAL SOLUTION}

In order to determine the phase velocity as a function of frequency for the various Lamb modes, we must solve Eq. (12) for the wave vector, $k$. Our approach has been to search the complex plane for approximate local minima of the determinant functior. The minimum is then located precisely using a two-axis Newton's approximation method; that is, the slope of the surface in both the real and imaginary directions is calculated using a step size of $10^{-10}$, and a weighted average is then taken to yield a conservative estimate of the location of the minimum. We found that a rather arcane collection of test statements was necessary to guarantee convergence to the minimum and to prevent the program from falling into an endless cycle of corrections which overshoot the minimum or that converge too slowly. In the final version, the program will generally locate the minimum of the surface within $10^{-8}$ along both the real and imaginary axes in ten steps (often in five steps, depending on the local nature of the surface). A final test determines if the minimum corresponds to a true root or merely to a nonzero minimum (which occurs, for example, when either the real or the imaginary part of the determinant, but not both, goes through zero). This approach will occasionally miss a root, but, in the event that the program is uncertain about the nature of a surface minimum, the complex plane location is printed so that the possible root can be examined in de tail with an interactive program.

In locating the roots of the secular determinant, we note that there is a branch point associated with each square root which defines the wave vectors $\alpha$ and $\beta$ [see Eq. (5), et seq.]. Thus, the roots of the secular equation will lie on several Riemann sheets. In defining the 
potentials as we have in Eq. (10), however, we see that the true Lamb modes (i.e., the real roots of the secular equation) should lie on the sheet corresponding to all positive branches of the square root functions. Leaky modes (not considered here) would occur on the sheet corresponding to the negative branch of the wave vector for the potential $\phi$ in the fluid in which the leaky wave propagates. The wave vectors $\alpha$ and $\beta$; the wave amplitudes $A_{1}, B_{1}, A_{2}$ and $B_{2}$; and the Lamb wave vector, $k$, all complex variables, are the quantities calculated and printed by our computer program.

The program to locate roots of the secular determinant is written in FORTRAN. In its original incarnation, the main routine would permit secular determinants up to 16 by 16 to be solved, which is sufficiently large to encompass a five-layer solid (three finite layers between two semi-infinite solids). This routine would then be linked with a subroutine called MATRIX, which defined the elements of the determinant whose roots were to be found. However, we have since found it more convenient to write a separate program for each class of guided wave to be studied. Thus, we now have, for example, a program called LAMB, which solves the 4 by 4 determinant given in Eq. (12). A second program, called LEAKLAMB and not considered here, solves the 6 by 6 determinant associated with leaky Lamb waves. Still other programs were written for Rayleigh, Love, Stoneley, and other guided wave types as well as for leaky waves of each of these types. All, however, were derived from the original generalized program written to solve the multilayer elastic guided wave problem.

\section{RESULTS}

The material properties of iridium alloy used in calculating the phase and group velocity dispersion curves were taken from an unpublished table compiled by $M$. W. Moyer of the Oak Ridge Y-12 Plant. The salient properties are: $C_{1}=5.305 \mathrm{~km} / \mathrm{s}, C_{t}=3.119 \mathrm{~km} / \mathrm{s}$, and $\rho=22,514 \mathrm{~kg} / \mathrm{m}^{3}$. Figure 3 shows the dispersion curves calculated for Lamb wave propagation in iridium alloy sheet. The ordinate of the graph gives the phase velocity $(C)$ of the Lamb wave normalized to that of a shear wave in iridium alloy. Since, by Snell's law, the phase velocity is related to the critical angle for Lamb wave generation from a surrounding fluid, the ordinate is also inversely proportional to the sine of the incident angle, the proportionality constant being the ratio of fluid velocity to iridium alloy shear wave velocity. The abscissa of the graph is also 
ORNL-DWG-94-10474

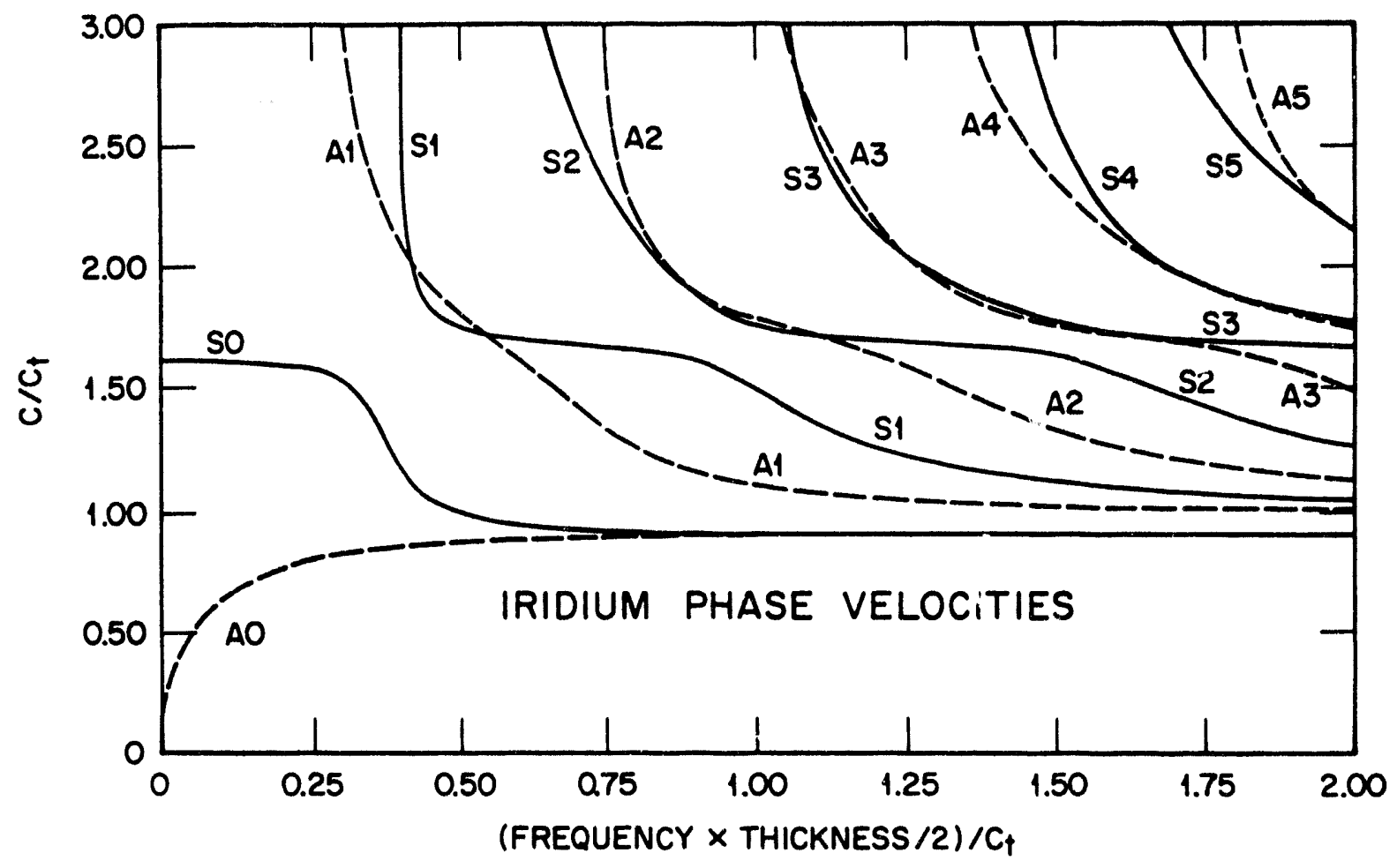

Fig. 3. Phase velocity dispersion curves showing Lamb wave modes in iridium alloy.

dimensionless, being the ratio of frequency times layer half-thickness to the shear velocity. By having dimensionless axes, Fig. 3 is applicable to any layer thickness.

Figure 3 shows a typical set of alternating symmetric (S) and antisymmetric (A) Lamb modes. As the frequency tends to zero, the only surviving mode is the lowest-order symmetric mode, $S_{0}$. For all other modes (except $A_{0}$, whose phase velocity asymptotically goes to zero in the low-frequency limit), there is a frequency limit below which the mode cannot propagate. The high-frequency limit of each mode, most easily seen for $S_{0}$ and $A_{0}$, is just the Rayleigh wave velocity. This is in agreement with intuition since in the high-frequency limit, the wavelength becomes negligible in comparison with the si.nple thickness, and the only guided wave that exists on the surface of a semi-infinite solid is a Rayleigh wave.

If one draws a horizontal line on Fig. 3 at some arbitrary ordinate value (corresponding to a particular phase velocity or incident angle), this line will intersect one or more of the possible Lamb modes. For example, assume that the Lamb wave is to be excited by a plane wave incident on the sample at an angle of $39^{\circ}$ in water. This incident angle, by Snell's law, will produce a Lamb wave whose phase velocity is $2.356 \mathrm{~km} / \mathrm{s}$, corresponding to an ordinate value of 0.756 in 
Fig. 3. A horizontal line drawn at this ordinat: will intersect only the $A_{0}$ mode. Moreover, the interection occurs at an abscissa value of 0.18 . For $0.635-\mathrm{mm}$-thick iridiun alloy, this value corresponds to a frequency of $1.76 \mathrm{MHz}$. Thus, using plane-wave excitation at a precise incident angle of $39^{\circ}$ should produce a single antisymmetric-mode Lamb wave in iridism alloy at the specified frequency. Even for plane-wave transducen, however, the finite element size will produce beamspread, leading to a small range of angles in the incident beam. Consequently, the $A_{0}$ mode Lamb wave will be excited over a range of frequencies, the extent of which depends on the range of angles in the incident beam and the slope of the mode response. If a focused transducer were used to excite this mode at the same centerline angle, the much greater range of angles present would probably excite the $A_{0}$ mode over the full bandwidth of the transducer, and the $S_{0}$ mode would likely be excited as well. On the other hand, Fig. 3 shows that, for ordinate values in the range 2 to 3 , the $S_{1}$ mode can be excited only over a very narrow range of frequencies because of the nearly vertical slope of the mode curve.

At an ordinate value of 1.5 (corresponding to an incident angle of $18.5^{\circ}$ ), Fig. 3 shows that six modes could be excited over the abscissa range depicted. These modes, in order of increasing frequency, are $S_{0} A_{1}, S_{1}, A_{2} S_{2}$ and $A_{3}$. Assuming the 0.635-mm thickness mentioned above, these modes would be excited at frequencies of 3.14, 6.58, 9.82, 13.3, 16.4, and $19.6 \mathrm{MHz}$, respectively.

Having obtained the phase velocities of the various Lamb modes, we next determined the group velocities. The slope of the phase velocity curve was determined numerically at a given frequency by taking a small step of 0.01 (or 0.001 when the slope was large) above and below the desired point and approximating the derivative by a two-sided difference. The step size was adjusted to yield at least six-figure accuracy for the approximation. The computed derivative, the frequency, and the phase velocity were then substituted in Eq. (16) and the group velocity determined.

Figure 4 shows the group velocity $\left(\mathrm{V}_{\ell}\right)$ dispersion curves for the first four Lamb modes. The results were limited to these modes because, at frequencies below about $10 \mathrm{MHz}$ in the iridium alloy sheet, Fig. 3 shows that only these modes can be generated. For mode $S_{0}$, the group velocity is equal to the phase velocity in the low-frequency limit but thereafter falls below the latter, reaching a minimum near an abscissa value of 0.39 . The high-frequency asymptotic limit is again the Rayleigh velocity. For mode $A_{0}$ the group velocity rises rapidly from its lowfrequency limit of zero and, at an abscissa value of about 0.33 , equals that of mode $S_{0}$. 
ORNL-DWG 94-10475

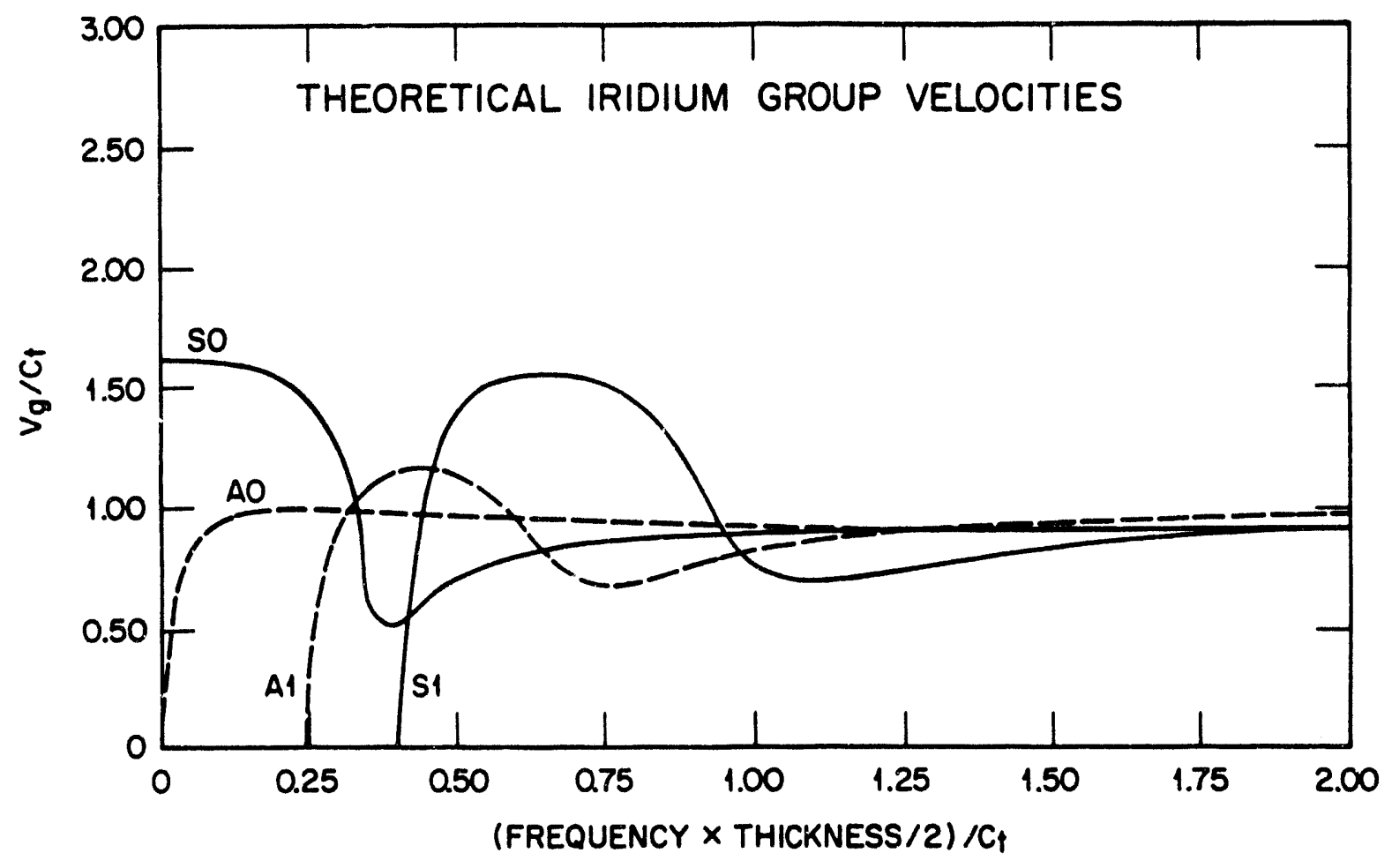

Fig. 4. Group velocity dispersion curves showing Lamb wave modes in iridium alloy.

Thereafter, $A_{0}$ is actually faster than $S_{0}$, in contrast with the results of Fig. 3. If one were to use the phase velocity information (as is often done) to predict the location of a flaw from its echo arrival time, the conclusions could be considerably in error, as Fig. 4 illustrates.

The results described above were confirmed in flat iridium alloy sheet. Two plane, 5-MHz, matched broadband transducers were used in the experimental studies. Figure 5 shows the rf waveform of one of the transducers and Fig. 6 the spectral content. These units have sufficient energy for useful measurements in the range -2 to $10 \mathrm{MHz}$. The transducers were mounted in a pitch-catch fixture, so that one unit acted as the transmitter and the other as receiver.

From Fig. 3 we see that, for ordinate values below about 0.92 (corresponding to an incident angle of $31.1^{\circ}$ ) and neglecting transducer beamspread, only the $A_{0}$ mode should be excited. Selecting an ordinate value of 0.75 , both transducers were set to an angle of $\sim 39^{\circ}$. Figure 7 shows the received if waveform and Fig. 8 its frequency content. The waveform, which approximates the cosine-modulated Gaussian mentioned earlier, suggests the presence of a single Lamb mode, which is confirmed by the spectrum. The peak of the spectral energy occurs at $1.9 \mathrm{MHz}$, which 
ORNL-DWG 94-10476

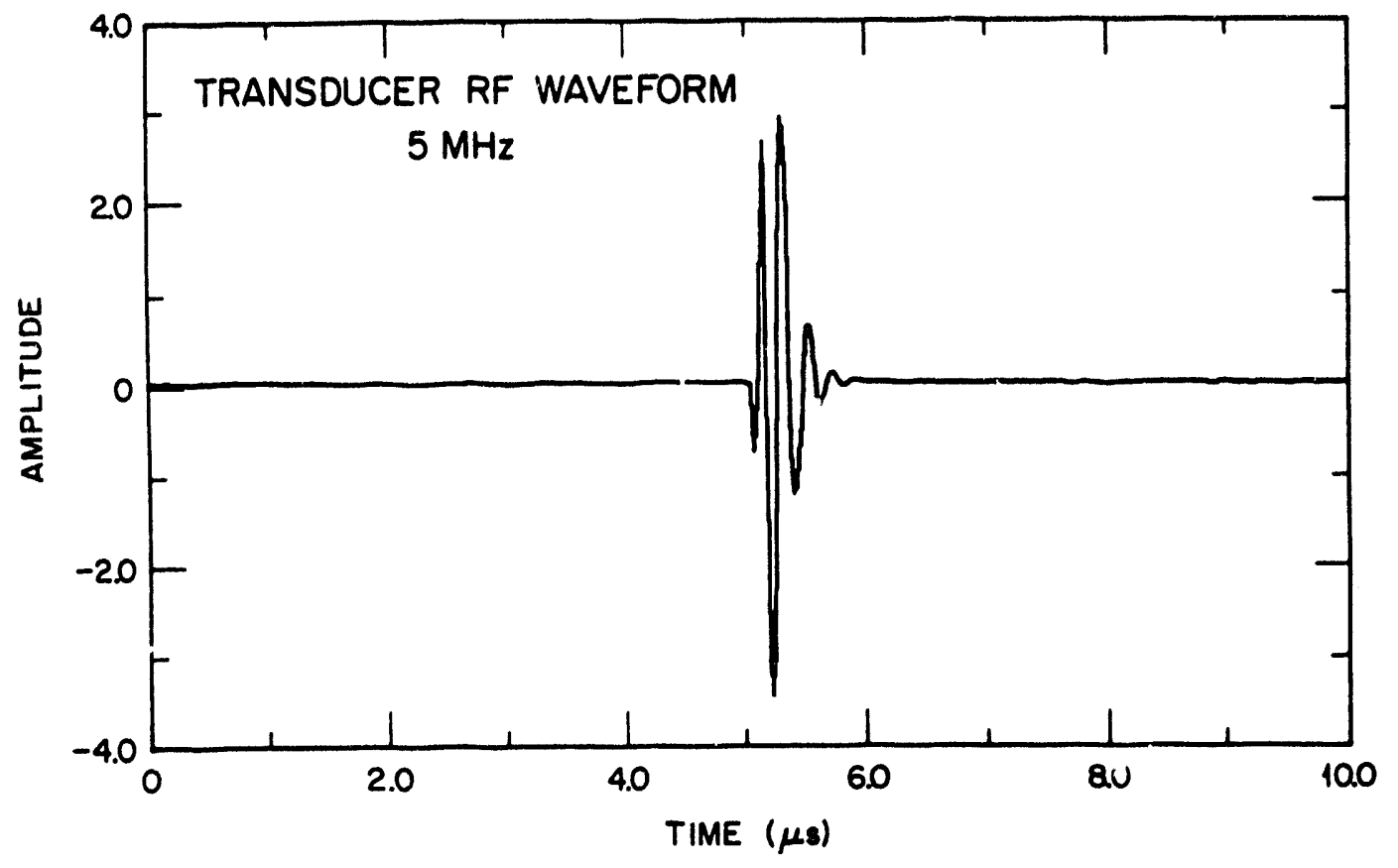

Fig. 5. Radio frequency waveform of transducers used to excite Lamb waves in iridium plate alloy.

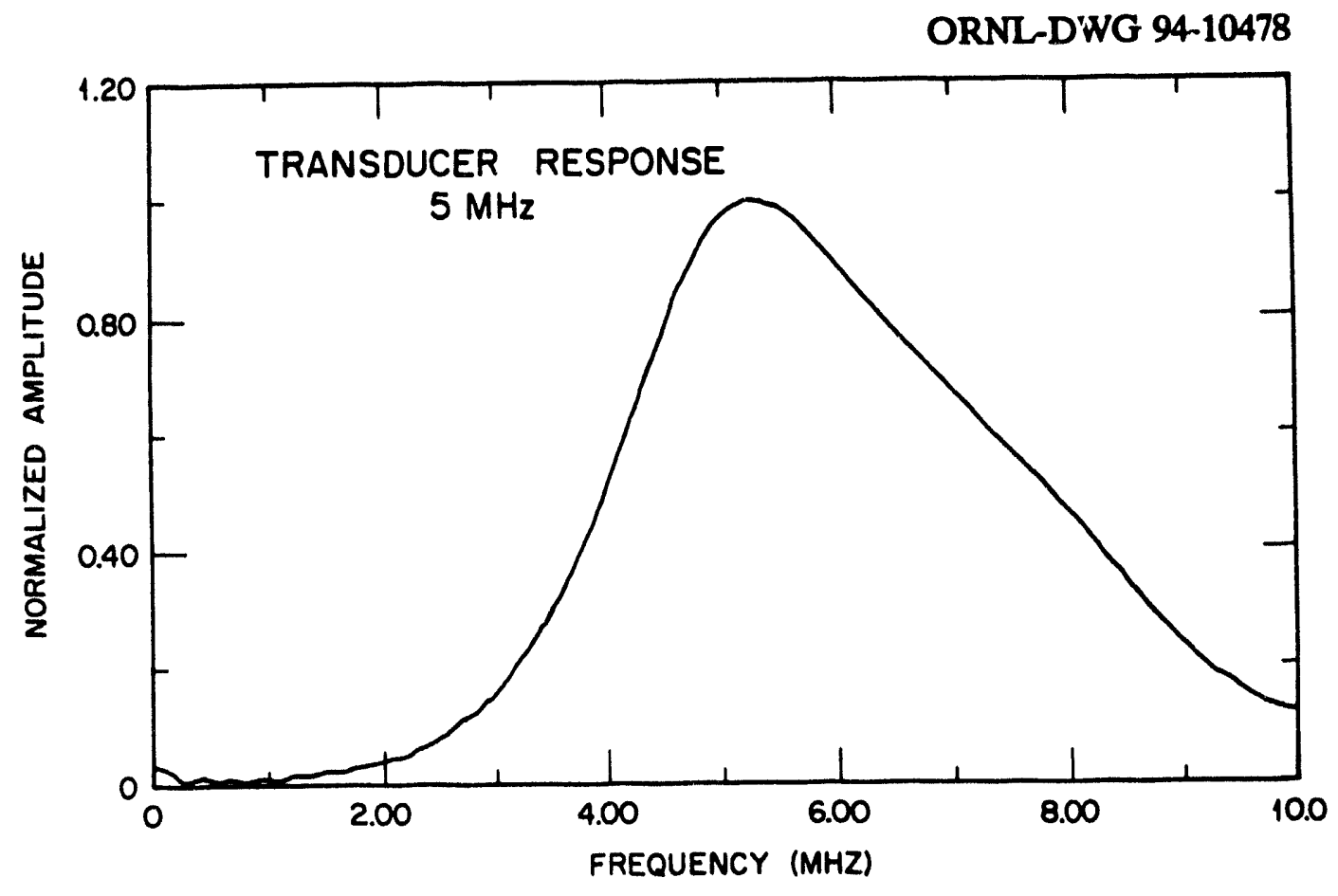

Fig. 6. Spectrum of transducers used to excite Lamb waves in an iridium plate alloy. 


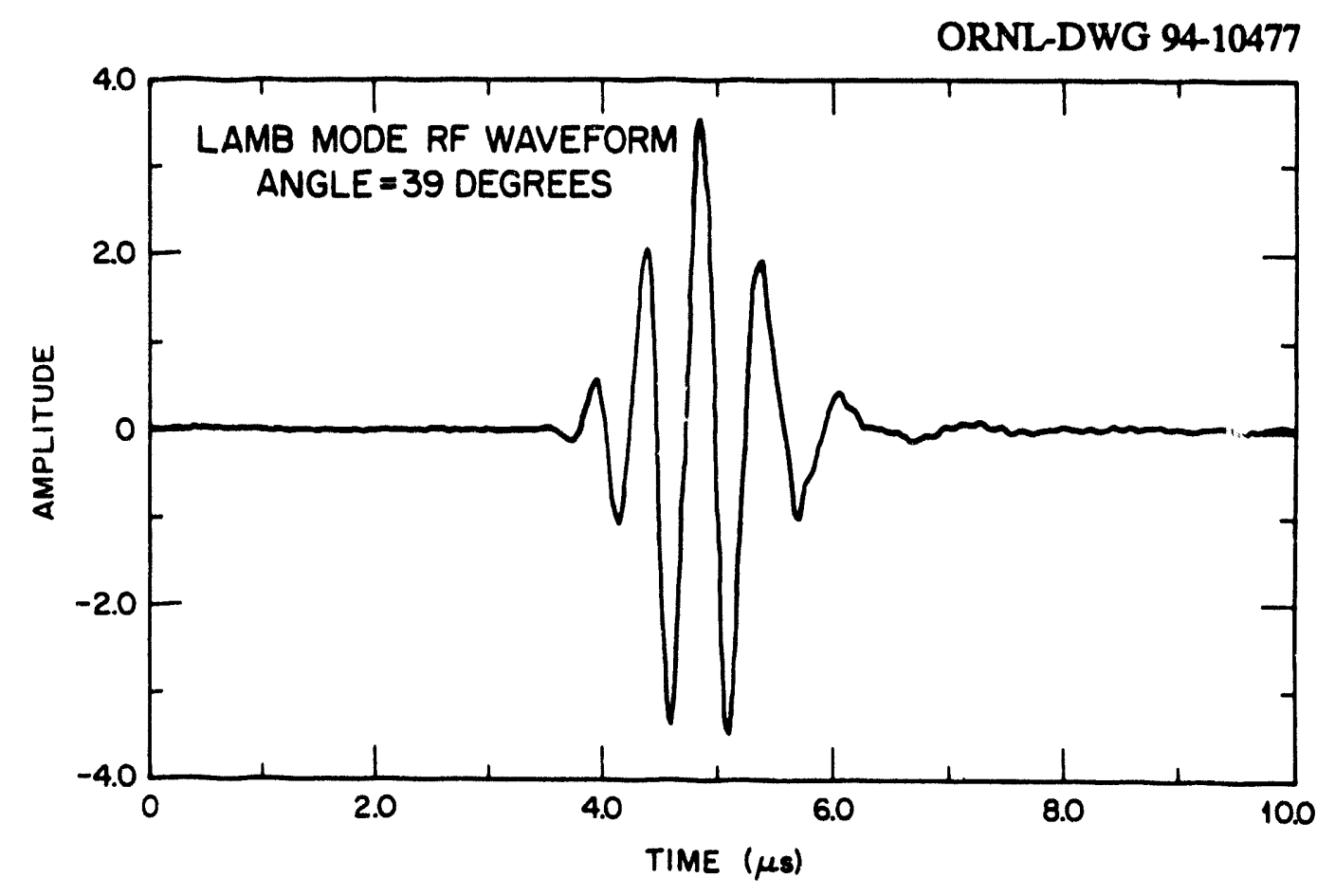

Fig. 7. Radio frequency waveform of $A_{0}$ Lamb mode in iridium alloy at $39^{\circ}$ incident angle.

ORNL-DWG 94-10479

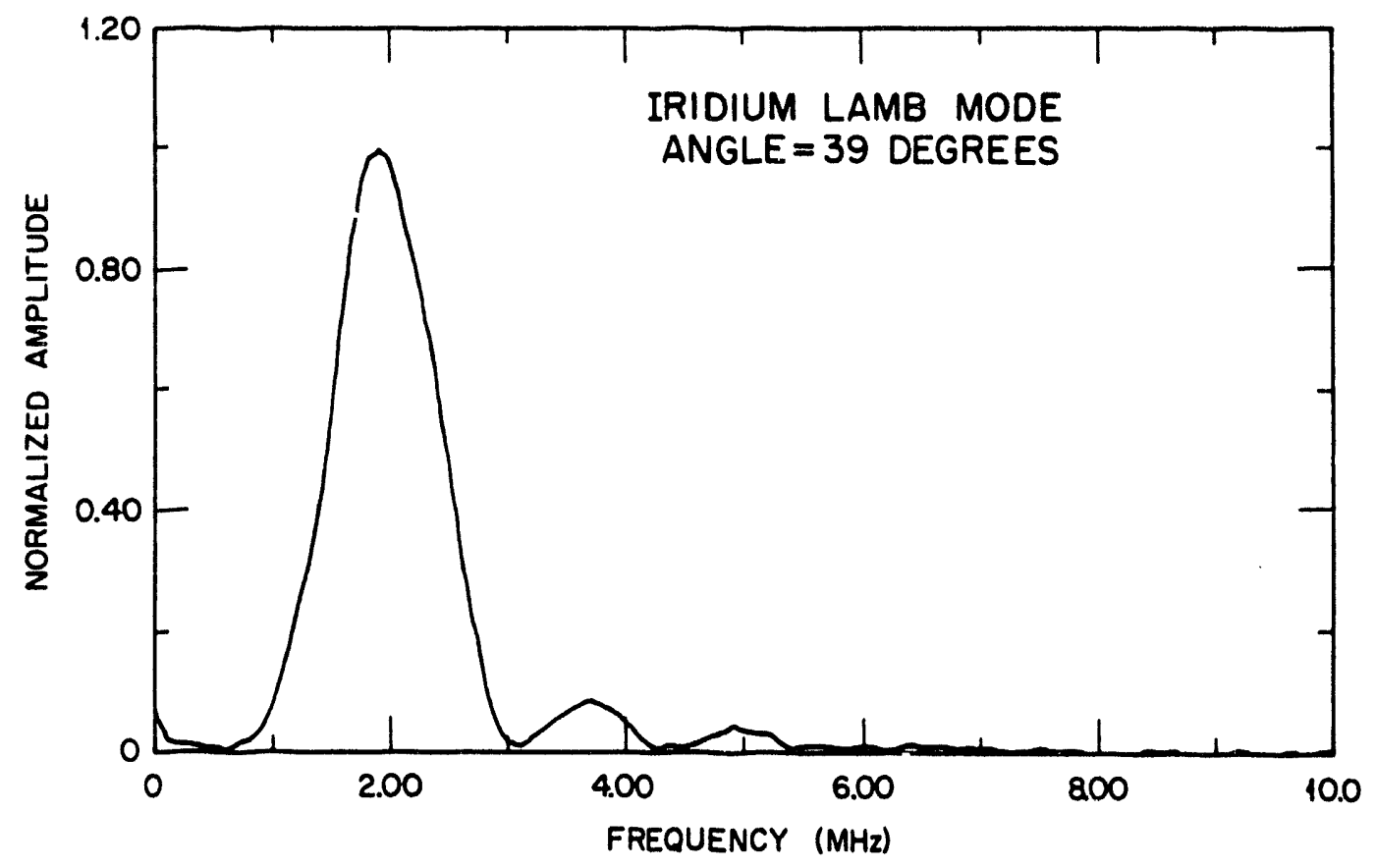

Fig. 8. Spectrum of $A_{0}$ Lamb mode in iridium alloy at $39^{\circ}$ incident angle. 
agrees well with the value of $1.8 \mathrm{MHz}$ predicted by Fig. 3. Some error is to be expected from unavoidable inaccuracies in the ultrasonic goniometers as well as from material properties which may differ slightly from the assumed values. The width of the spectral envelope is determined both by transducer beamspread and by the relatively small slope of the $A_{0}$ curve near the intersection point.

At an ordinate value of 1.5 (incident angle $18.5^{\circ}$ ), Fig. 3 indicates that three modes will be intercepted in the region of abscissa values less than or equal to $1\left(S_{0}\right.$ at $3.1 \mathrm{MHz}, A_{1}$ at $6.6 \mathrm{MHz}$, and $S_{1}$ at $9.8 \mathrm{MHz}$ ). Figure 9 shows the if waveform of the received Lamb waves and Fig. 10 their spectrum. As expected, the latter figure shows that three waves are present. The spectral peaks of the two higher-frequency modes occur at frequencies lower than predicted, but this is likely attributable to errors in the measurement of the incident angle. This is reinforced by the fact that subsequent spectral peaks invariably occurred at slightly lower than predicted values, irdicating a systematic error typical of goniometer inaccuracy.

Figure 9 also illustrates a problem mentioned earlier. Thire are three wave packets in this composite waveform (corresponding to the three generated Lamb modes), and it would be impossible to measure the group velocity of any of the three from such an $\mathrm{rf}$ response. On the other hand, Fig. 10 shows that the modes are well resolved in the frequency domain, and hence measurements carried out here should be free of mutual interference. That the wave packets should arrive at approximately the same time is supported by the predictions of Fig. 4, where the group velocities of the three waves are nearly equal at the appropriate abscissa values, with $\mathrm{S}_{0}$ having a slightly higher velocity than the other two modes.

For an ordinate value of 2.5, Fig. 3 indicates that four modes, $A_{1}, S_{1}, S_{2}$ and $A_{2}$, should be generated in the range 2 to $10 \mathrm{MHz}$ (abscissa values 0.2 to 1.02). These waves occur at an incident angle of about $11^{\circ}$. In addition, Fig. 3 indicates that the slopes of the curves $S_{1}$ and $A_{2}$ are nearly vertical at this ordinate value; hence, these waves should be nearly monochromatic. This is confirmed by toe experimental data. Figure 11 shows the received composite waveform and Fig. 12 the spectrum. Because of the limited memory length in our digitizing oscilloscope, the time-domain record was inadequately sampled. Thus, the nearly monochromatic peaks near $4 \mathrm{MHz}\left(S_{1}\right)$ and $7.4 \mathrm{MHz}\left(A_{2}\right)$ in Fig. 12 are not well represented. An analog spectrum analyzer confirmed the presence of these very sharp peaks, however. The broader peaks at about 3.6 and $7 \mathrm{MHz}$ belong to $A_{1}$ and $S_{2}$, respectively. Neither $S_{2}$ nor $A_{2}$ would likely be present during capsule inspection unless the exciting transducer had reasonable response at frequencies at and 
ORNL-DWG 94-10480

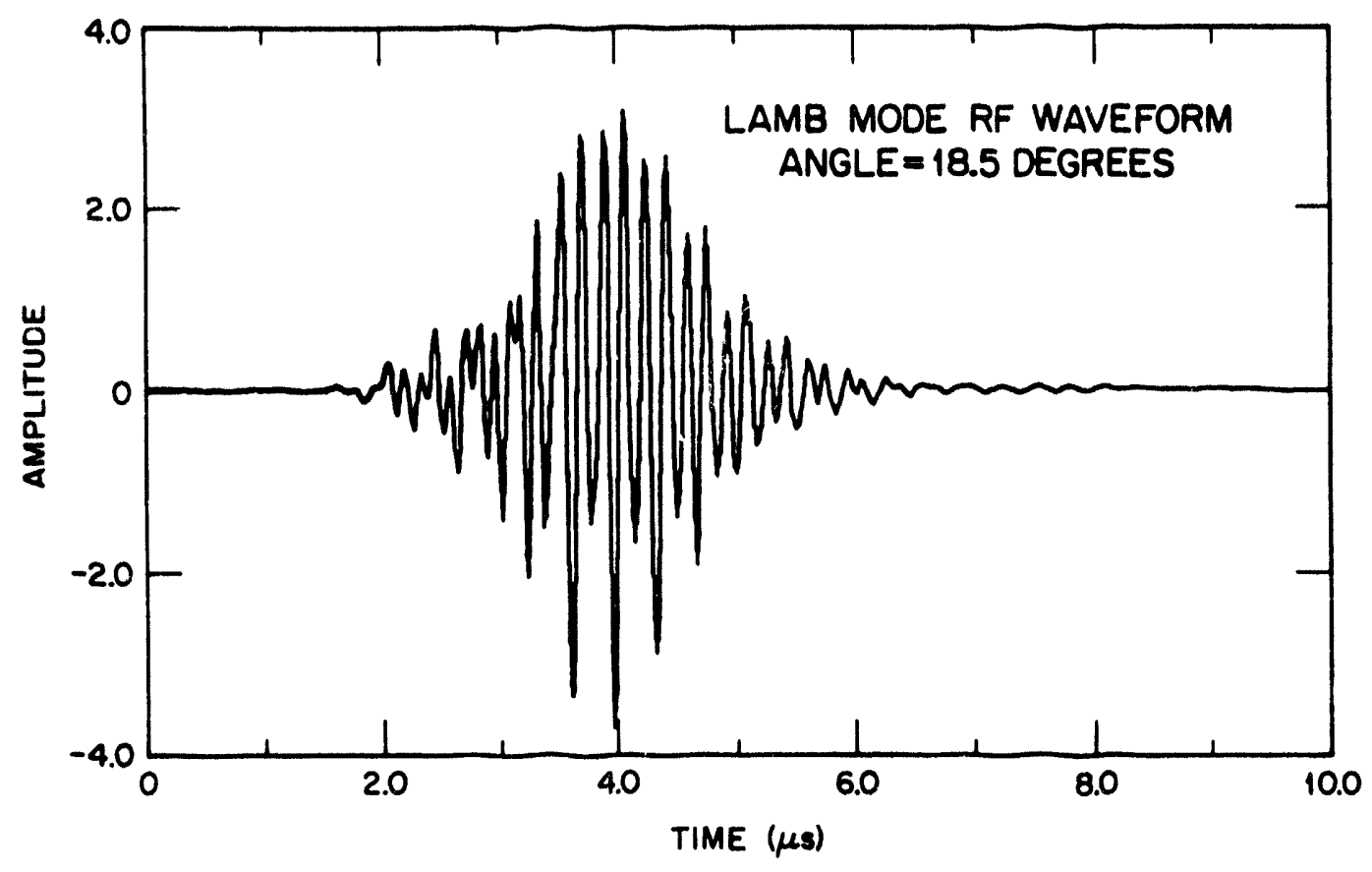

Fig. 9. Radio frequency waveform of combined $S_{0}, A_{1}$, and $S_{1}$ Lamb modes in iridium alloy at $18.5^{\circ}$ incident angle.

ORNL-DWG 94-10481

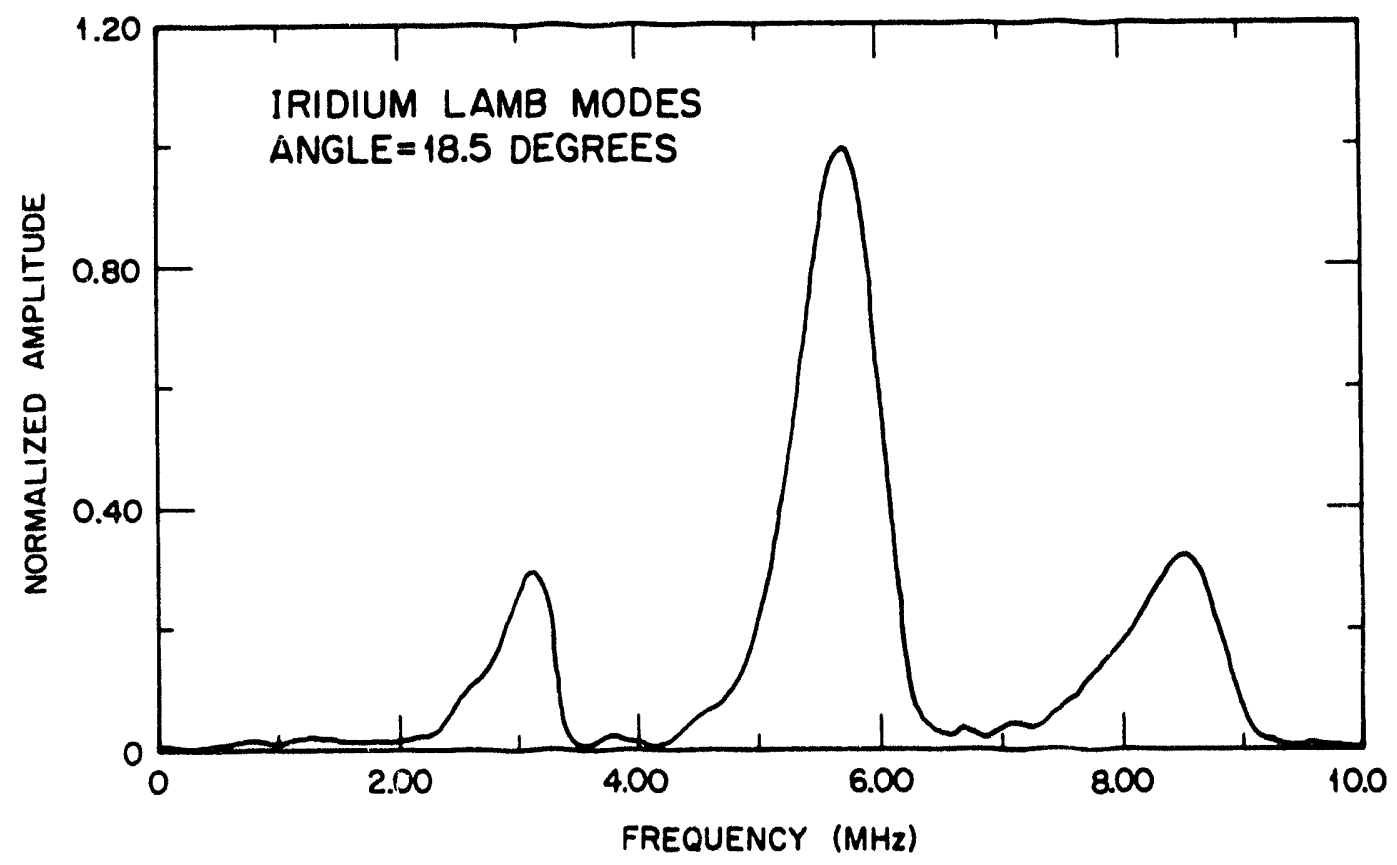

Fig. 10. Spectrum of $S_{0}, A_{1}$, and $S_{1}$ Lamb modes in iridium alloy at $18.5^{\circ}$ incident angle. 
ORNLDWG 94-10482

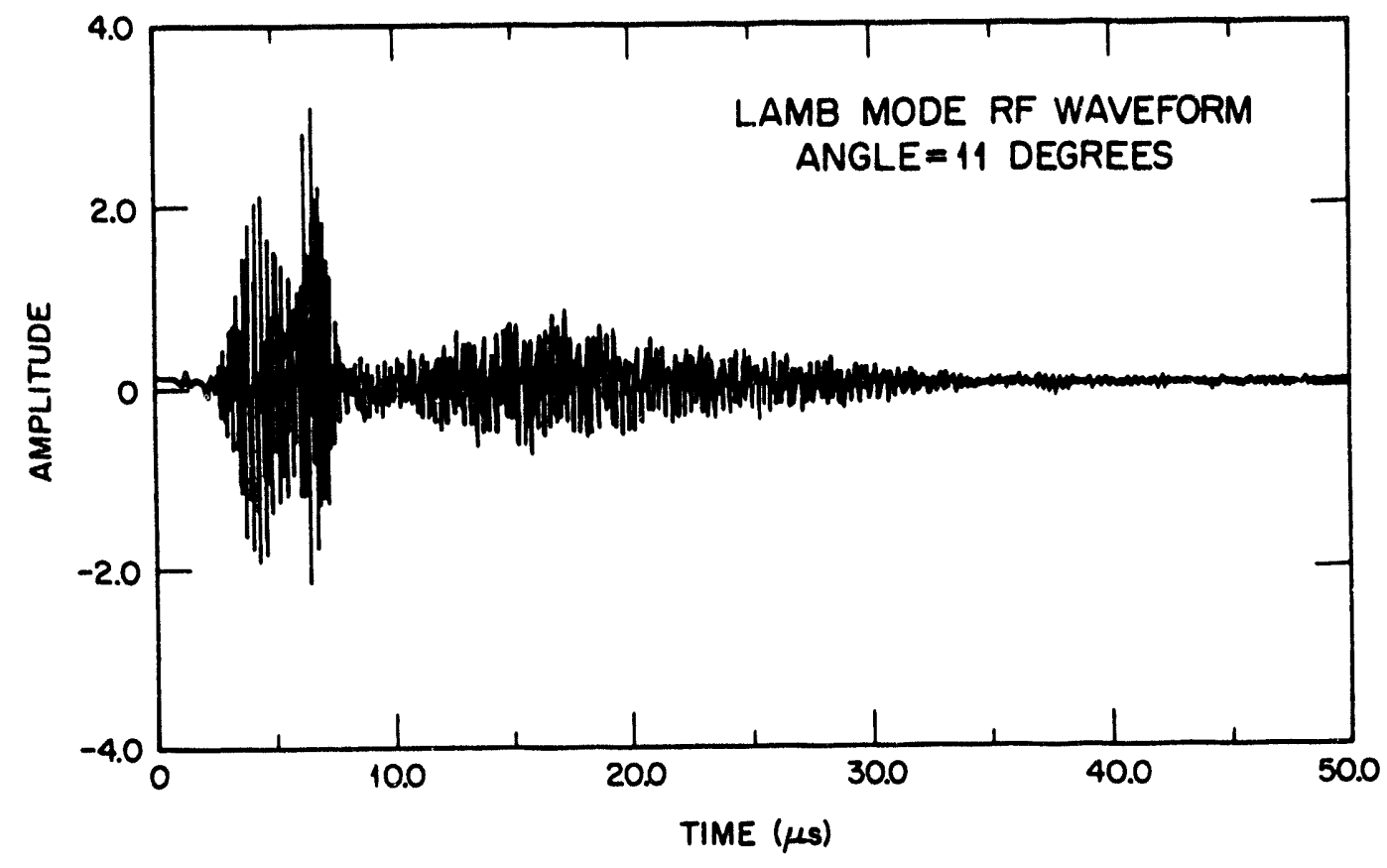

Fig. 11. Radio frequency waveform of combined $A_{1}, S_{1}, A_{2}$ and $S_{2}$ Lamb modes in iridium alloy at $11^{\circ}$ incident angle.

ORNL-DWG 94-10483

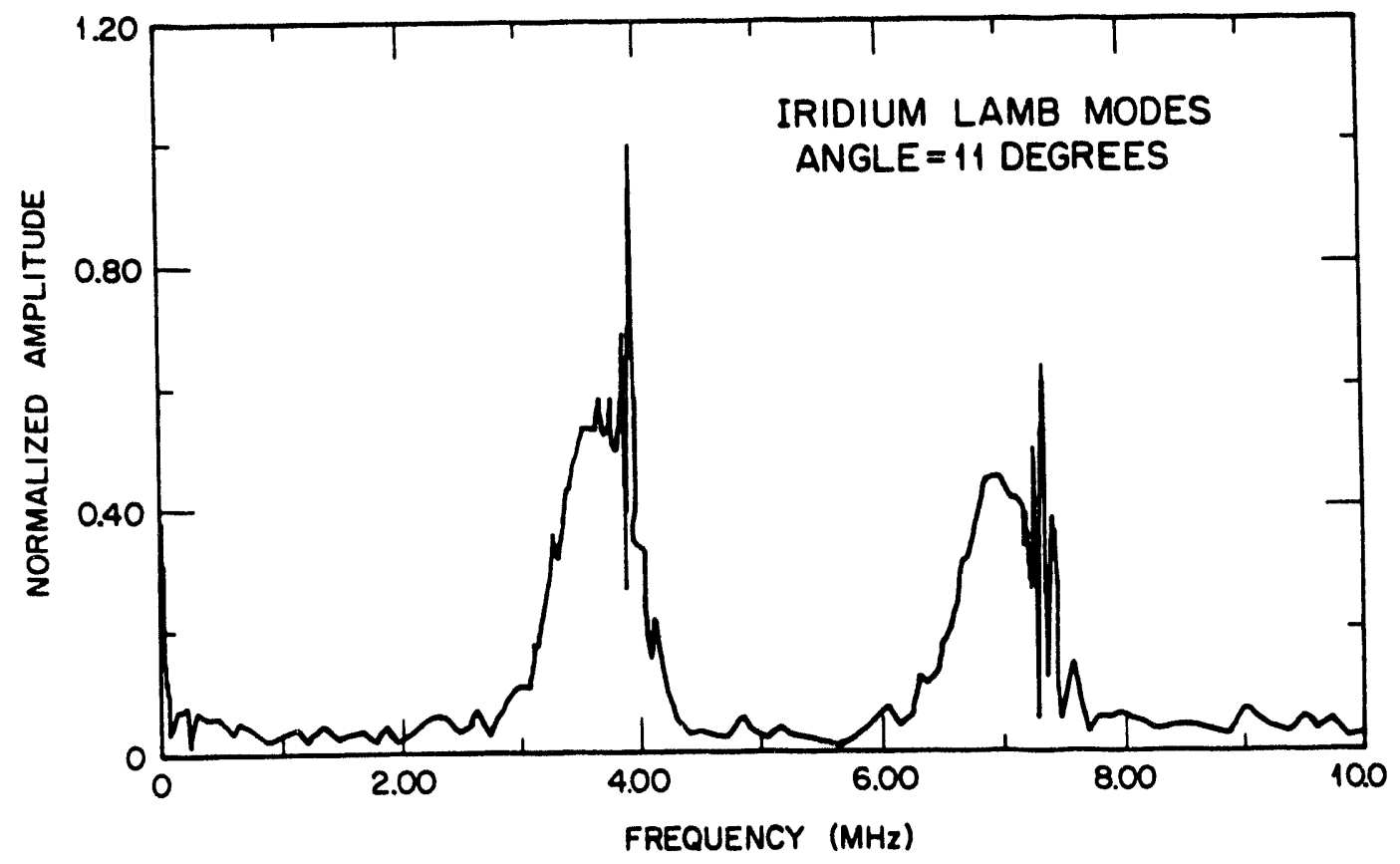

Fig. 12. Spectrum of $A_{1}, S_{1}, A_{2}$, and $S_{2}$ Lamb modes in iridium alloy at $11^{\circ}$ incident angle. 
above $8 \mathrm{MHz}$; for this reason, neither of these modes was included in the group velocity curves of Fig. 4.

Because two quasimonochromatic waves are present at an incident angle of $11^{\circ}$, the wave packets of these two Lamb modes will be quite extended in time. This is admirably illustrated in Fig. 11, where a time-domain record $50 \mu s$ in length was required to capture most of the wave train. The ringing beyond about $10 \mu \mathrm{s}$ is caused almost exclusively by $S_{1}$ and $A_{2}$ while the peaks near $5 \mu$ are caused by the more broadband modes $A_{1}$ and $S_{2}$. The figure also shows that the group velocities of $S_{1}$ and $A_{2}$ are slower than those of $A_{1}$ and $S_{2}$, whose wave packets arrive earlier. This information can also be gleaned from Fig. 4, at least for $A_{1}$ and $S_{1}$.

These results indicate that, using the data of Fig. 3, the investigator can easily predict which Lamb modes will be present for a given incident angle, the frequencies at which these modes will occur, and, at least qualitatively, what the relative mode bandwidths will be. For accurate flaw location, however, it is the group velocity (Fig. 4) that is important, and a measurement of this quantity must be addressed.

As we mentioned earlier, because of the presence of multiple Lamb modes at most incident angles, accurate measurements of the group velocity must be performed in the frequency domain using phase-slope methods [Eq. (19)]. To implement this technique, we computed the phase spectrum using the FFT. Two transducers in a pitch-catch arrangement were used, and the path length was changed by a known amount, following which the FFT was recomputed. The change in the phase-slope characteristic over the known differential path length was then determined and the group velocity computed using Eq. (19). Several error sources for this approach were recognized. Chief among these is the goniometer error in determining the incident angle, as mentioned earlier. In addition, the limited memory length of our digitizing oscilloscope required a relatively low sample rate for long record lengths, which in turn caused a relatively large time-domain error. In most cases, however, the results were adequate to validate the theoretical predictions.

Examination of Figs. 3 and 4 also suggests potential problem areas. For the mode $A_{0}$, for example, the group velocity (Fig. 4) increases very rapidly near the origin. This means that, even for small errors in the incident angle, the relative error in group velocity can be large. This same reasoning applies to any mode near its asymptotic limit. In addition, for $\mathrm{A}_{0}$ the region in which this rapid increase occurs lies in the range 0 to $1 \mathrm{MHz}$, where the exciting transducers have very little energy (Fig. 6). Near the Rayleigh velocity (ordinate value -0.92 in Fig. 3), modes $A_{0}$ and 
$S_{0}$ coalesce and cannot be separated even in the frequency domain (the group velocities, however, remain quite disparate in the range of abscissa values -0.34 to 0.7 in Fig. 4). For modes $A_{1}$ and $S_{1}$, Fig 3 shows that, in the range of abscissa values -0.4 to 0.6 , the modes are too closely spaced in the frequency domain for proper separation. In the range of ordinate vlues $>2.5$, the group velocities of these same modes are approaching their asymptotic limits and are again difficult to measure. In summary, difficulties may be expected to arise whenever we approach too closely the asymptotic limit of a mode, or when two or more modes are inadequately separated in both time and frequency domains.

In measuring group velocity, we followed the procedure suggested by Eq. (19); i.e., we determined the slope of the phase characteristic after propagation through a known distance and computed the group velocity using the equation. Figure 13, which is a repeat of the theoretical curves of Fig. 4 with our measured values superimposed, shows the results. We were primarily interested in verifying the shapes of modes $A_{1}$ and $S_{0}$ since these modes will probably be most important in evaluation of the capsules. Figure 13 also shows that, except for a single data point, all of the measured velocities fell slightly below the predicted values. As mentioned earlier, this is a systematic error which is probably attributable to errors in the goniometer or to slight differences in the assumed and actual values for the material properties of the iridium alloy. The one exception occurred for mode $S_{1}$, where the data point was probably affected by some contamination from mode $A_{1}$ (Fig. 3).

\section{SUMMARY AND CONCLUSIONS}

The secular determinant for Lamh .....es propagating in a plate was derived and applied to the specific case of iridium alloy sheet. A computer program was written which finds the roots, both real and complex, of this determinant, and the roots for 0.64 -mm-thick alloy sheet were determined. From these roots, a graph of the phase velocity as a function of frequency was constructed for the various Lamb modes. A method for computing the group velocity of any mode from the phase velocity characteristic was presented, and a graph of the group velocities of the first four Lamb modes was constructed.

Because of the difficulty of measuring group velocity in the time domain when two or more Lamb waves are present simultaneously, a frequency-domain technique that relies on the phaseslope behavior of the mode was presented and applied. Group velocities were measured at several points on the dispersion curves for the first four Lamb modes. 


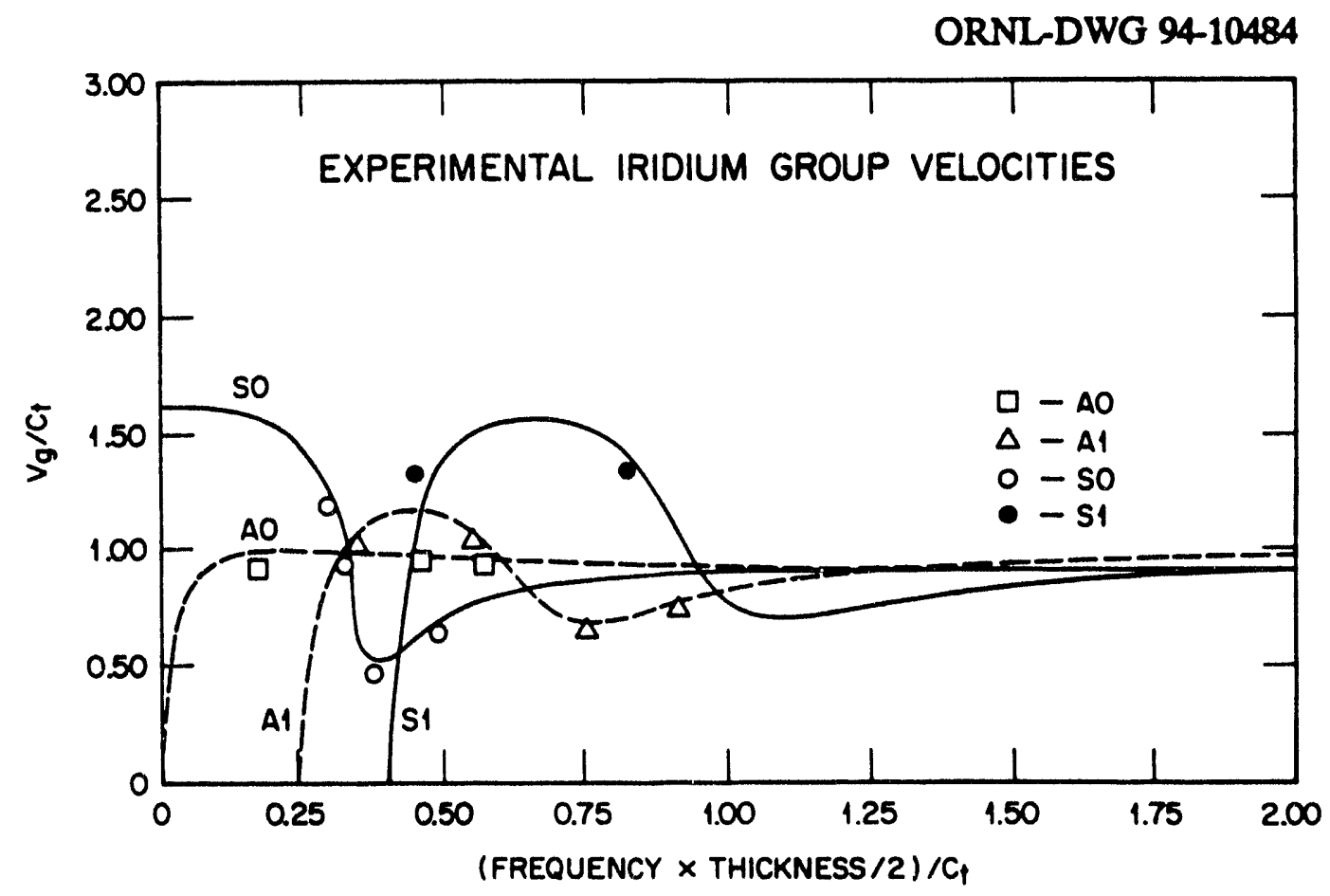

Fig. 13. Measured values of group velocity for four Lamb modes in iridium alloy.

A study of the possibility of predicting the time-domain behavior of Lamb waves was undertaken. Because of the complexity of the problem and the limited time available for the study, the results were restricted to analytical expressions for dispersive plane waves, a simplification that appears to yield surprisingly good results for some cases. A general treatment of this topic would need considerably more time, however, but the preliminaries are in place for a resumption of this effort if the results are deemed necessary.

Because of the material properties (primarily grain size) of iridium alloy, Lamb waves are necessarily generated by the low inspection frequencies required to minimize grain boundary scattering losses in the thicknesses chosen for clad vent set capsules. This study of Lamb wave propagation in the alloy has shown that, insofar as facilitating the interpretation of inspection results is a primary goal, the researcher can achieve this goal by choosing a test configuration that provides maximum resolution of the various modes in the inspection domain. That is, if the inspection is conducted in the frequency domain, then the operating conditions should be chosen to provide maximum frequency separation of all modes which may be present. On the other hand, if the inspection is carried out in the time domain, then the proper choice would be that which maximizes the disparity in the group velocities of the various modes. In this manner, the 
investigator can be certain which ones of the possible modes are actually contributing to the acquired data. In addition, the manner in which ultrasound is introduced into the capsule is critically important in determining the complexity of the resulting ultrasonic data display. If the incident energy is introduced in the knuckle region of the capsule using a focused transducer, for instance, essentially all incident angles from 0 to $90^{\circ}$ will be present, and the resulting display will be very complex and consist of combinations of several Lamb modes. In addition, very little of the available energy would propagate in the desired direction (i.e., along the capsule axis). A better configuration would be that in which the ultrasonic energy is introduced in the flat region on the capsule top using the same transducer. The range of incident angles would be greatly reduced, thereby simplifying the Lamb mode spectrum, but much of the energy would still propagate in nonuseful directions. Perhaps the best configuration would be that in which a planewave transducer was used to generate a single Lamb mode in the capsule top flat region. With this configuration, the researcher could be assured of precisely which mode was generated (indeed, the mode generated could be controlled by changing the incident angle). Lastly, this configuration would produce maximum energy in the desired direction, thereby increasing test sensitivity.

Regardless of which configuration is used in evaluating the capsules ultrasonically, the results of this study will allow the investigator to interpret the test results more clearly and quickly. In particular, the group velocity curves will permit identification of the Lamb mode producing a given flaw signal and yield more accurate flaw locations by providing exact group velocity information.

\section{ACKNOWLEDGMENTS}

The authors would like to thank W. J. Barnett of the Department of Energy, Office of Nuclear Energy, Radioisotope Power Systems Division, for his support of this study. Thanks are also due to M. W. Moyer of the Oak Ridge Y-12 Plant for providing the elastic properties of iridium alloy and for elucidation of the test configurations used in inspecting assembled capsules, and to K. V. Cook of Oak Ridge National Laboratory for his help and suggestions. The authors also thank K. Spence for editing, G. R. Carter for quality assurance review, and Donna Balltrip for final report preparation. 


\section{REFERENCES}

1. J. Wolf, T. D. K. Ngoc, R. Kille, and W. G. Mayer, "Investigation of Lamb Waves Having a Negative Group Velocity," J. Acoust. Soc. Am. 81(1), 122-26 (1988).

2. H. Lamb, "On Waves in an Elastic Plate," Proc. R. Soc. London, A 93, 114-28 (1917).

3. J. D. Achenbach, Wave Propagation in Elastic Solids, North-Holland, Amsterdam, 1980.

4. W. A. Simpson, Jr., and R. W. McClung, "An Investigation of Elastic Guided Waves for Ceramic Joint Evaluation, " ORNL/TM-10931, Martin Marietta Energy Systems, Inc., Oak Ridge Natl. Lab., October 1989.

5. D. E. Chimenti and S. I. Rokhlin, "Influence of Fluid Loading on Reflection' fficient Zeroes and Leaky Lamb Modes for a Composite Plate," Riv. Prog. Quant. Nondest. Eval 10A, 209-16 (1991).

6. R. Truell, C. Elbaum, and B. Chick, Ultrasonic Methods in Solid State Physics, Academic Press, New York, 1969.

7. W. Sachse a:d Y. Pao, "On the Determination of Phase and Group Velocities of Dispersive Waves in Solids", J. Appl. Phys. 49(8), 4320-27 (1978).

8. J. D. Jackson, Classical Electrodynamics, John Wiley and Sons, New York, 1962. 
ORNL/TM-12749

\section{INTERNAL DISTRIBUTION}

1-2. Central Research Library

3. Document Reference Section

4-5. Laboratory Records Department

6. Laboratory Records, ORNL RC

7. ORNL Patent Section

8-10. M\&C Records Office

11. R. H. Cooper, Jr.

12. D. F. Craig

13. H. W. Hayden, Jr.

14. K. J. Kozaczek

15-19. M. M. Martin

20-24. D. J. McGuire
25. G. E. Michaels

26. J. P. Moore

27-31. M. W. Moyer

32. E. K Ohriner

33. M. J. Saltmarsh

34-38. W. A. Simpson, Jr.

39. G. B. Ulrich

40. X. Wang

41. H. W. Foglesong (Consultant)

42. E. L Menger (Consultant)

43. I. G. Simon (Consultant)

44. K. E. Spear (Consultant)

\section{EXTERNAL DISTRIBUTION}

45. AMES LABORATORY, 242 Spedding Hall, Iowa State University, Ames, IA 50011 B. A. Cook

46-47. AUBURN UNIVERSITY, Materials Engineering Depertment, Auburn, AL 36849
A. N. Gubbi
R. H. Zee

48. BATTELLE COLUMBUS OPERATIONS, 505 King Ave., Columbus, OH 43201

C. A. Alexander

49. EG\&G MOUND APPLIED TECHNOLOGIES, INC., P.O. Box 3000, Miamisburg, OH 45342

W. R. Amos

50-51. FAIRCHILD SPACE COMPANY, 20301 Century Blvd., Germantown, MD 20874

R. T. Carpenter

E. A. Skrabek 
52. JET PROPULSION LABORATORY, California Institute of Technology, 4800 Oak Grove Drive, Pasadena, CA 91103

R. W. Campbell

53-54. JOHNS HOPKINS UNIVERSITY, Applied Physics Laboratory, Johns Hopkins Road, Laurel, MD 20723

J. C. Hagan

E. F. Lucero

55-56. LOS ALAMOS NATIONAL LABORATORY, P.O. Box 1663, Los Alamos, NM 87545

E. M. Foltyn

T. G. George

57-58. MARTIN MARIETTA ASTRO SPACE, P.O. Box 8555, Philadelphia, PA 19101

R. J. Hemler

R. M. Reinstrom

59. NUS CORPORATION, 910 Clopper Road, Gaithersburg, MD 20878-1399

B. W. Bartram

60. PHILLIPS LABORATORY, Kirtland Air Force Base, NM 87117

M. J. Schuller

61-62. ROCKWELL INTERNATIONAL, Rocketdyne Division, 6633 Canoga Avenue, Canoga Park, CA $91309-7922$

L. J. Auge

E. B. Baumeister

63. TELEDYNE ENERGY SYSTEMS, $110 \mathrm{~W}$. Timonium Road, Timonium, MD 21093 W. M. Brittain

64. WESTINGHOUSE ADVANCED TECHNOLOGY BUSINESS AREA, Energy Center East 310, Pittsburgh, PA 15230

M. O. Smith 
65-68. WESTINGHOUSE SAVANNAH RIVER COMPANY, Savannah River Site, Aiken, SC 29808
E. A. Franco-Ferriera
A. Plact
R. W. Saylor
W. D. Stephens

69. DEPARTMENT OF ENERGY, Office of Nuclear Energy, NE-50, GTN, Washington, D.C. 20585
A. R. Newhouse

70.82 DEPARTMENT OF ENERGY, Office of Nuclear Energy, NE-53, GTN, Washington, D.C. 20585
W. J. Barnett (9)
B. A. Cook
R. G. Lange
E F. Mastal
A. S. Mehner

83. DEPARTMENT OF ENERGY, Albuquerque Operations Office, P.O. Box 5400, Albuquerque, NM 87115
R. L. Holton

84. DEPARTMENT OF ENERGY, Dayton Area Operations Office, P.O. Box 66, Miamisburg, OH 45342

T. A. Frazier

85. DEPARTMENT OF ENERGY, Los Alamos Area Office, 528 35th Street, Los Alamos, NM 87544

R. J. Valdez

86. DEPARTMENT OF ENERGY, Savannah River Operations Office, Bldg. 703F, P.O. Box A, Aiken, SC 29802

S. W. McAlhaney

87. DEPARTMENT OF ENERGY, Oak Ridge Operations Office, P.O. Box 2008, Oak Ridge, TN 37831

S. R. Martin, Jr. 
88-89. DEPARTMENT OF ENERGY, Oak Ridge Operations Office, P.O. Box 2009, Oak Ridge, TN 37831

P. A. Carpenter

R. L. Lahti

90. DEPARTMENT OF ENERGY, Oak Ridge Operations Office, P.O. Box 2001, Oak Ridge, TN 37831

Assistant Manager, Energy Research and Development

91-92. DEPARTMENT OF ENERGY, Office of Scientific and Technical Information, Office of Information Services, P.O. Box 62, Oak Ridge, TN 37831

For distribution by microfiche as shown in DOE/OSTII-4500, Distribution Category UC-506 (Instruments, Engineering and Equipment). 

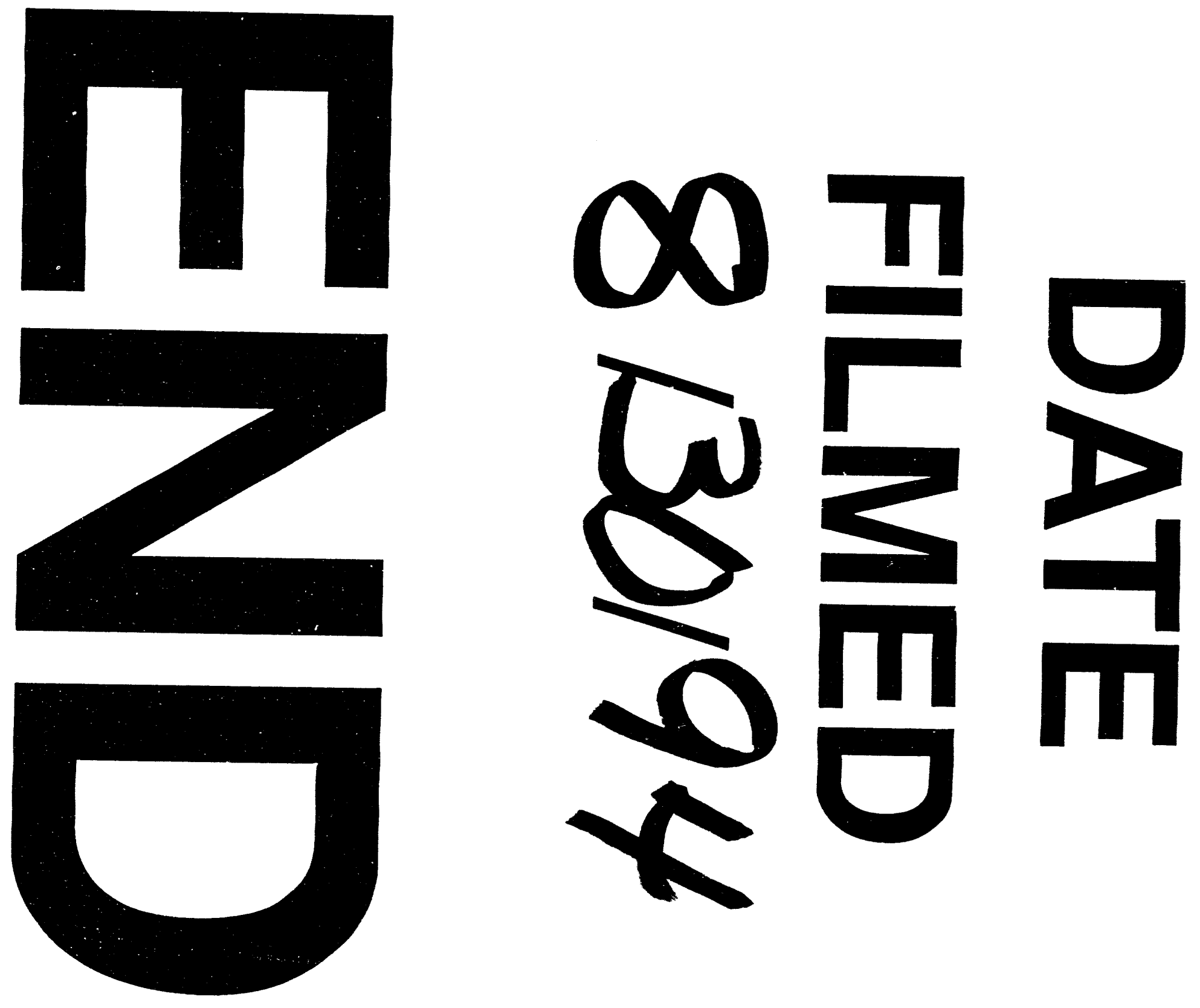
. 